\title{
La estela de Rediukhnum de Dendera y la reorganización administrativa del Estado egipcio a finales del III milenio a.C.*
}

\author{
4 Daniel González León** \\ Universitat Autònoma de Barcelona
}

Fecha de recepción: 7 de julio de 2018. Fecha de aceptación: 1 de agosto de 2018.

\begin{abstract}
Resumen
El proceso de reorganización administrativa que condujo a la formación del Estado egipcio del Reino Medio tuvo lugar a principios de la XI dinastía. La estela de Rediukhnum de Dendera es una de las fuentes epigráficas más importantes de que se dispone para reflexionar sobre este proceso. A través de un estudio filológico y paleográfico del texto, de un análisis de los títulos de Rediukhnum y de un estudio comparativo con otras fuentes contemporáneas o de épocas cercanas, se puede proponer una datación de la estela y deducir las medidas administrativas que estos primeros reyes tebanos adoptaron en las provincias más meridionales del Alto Egipto a finales del Primer Periodo Intermedio.
\end{abstract}

\begin{abstract}
The process of the administrative reorganization which resulted in the formation of the Egyptian state of the Middle Kingdom took place at the beginning of the 11th Dynasty. The stela of Rediukhnum of Dendera is one of the most important epigraphic resources available which reflects on this process. Through philological and paleographical research on the text, an analysis of the titles of Rediukhnum, and a comparative survey with other contemporary or closely dated resources, a date for the stela can be proposed. In addition, the administrative measures which these early Theban kings took in the southernmost provinces of Upper Egypt at the end of the First Intermediate Period can be deduced.
\end{abstract}

\footnotetext{
* Me gustaría expresar mi agradecimiento al Dr. Josep Cervelló Autuori (Universitat Autònoma de Barcelona) por su orientación y asesoramiento en la preparación del presente artículo.

*** Beneficiario del Programa Predoctoral de Formación de Personal Investigador No Doctor del

Departamento de Educación del Gobierno Vasco
}

Palabras clave

Rediukhnum XI dinastía Dendera administración provincial
Keywords

Rediukhnum 11th dynasty Dendera provincial administration 
1. El cementerio de Dendera fue excavado primero por W.M.F. Petrie y después por C. Fisher, de la Universidad de Pennsylvania. En la actualidad, la mayor colección de hallazgos procedentes de

Dendera se encuentra en el Museo de El Cairo y en el Museo de la Universidad de Pennsylvania.

2. Es una estela de piedra caliza de grandes dimensiones (152x62 cms.).

Consta de dos registros: el registro superior abarca casi toda la pieza y contiene una inscripción de 23 líneas, las cuales son de lectura complicada debido al mal estado de conservación de la pieza; y el registro inferior presenta a la izquier-

da a Rediukhnum sentado y con una jarrra en sus manos, y a la derecha a 4 de sus sirvientes en acto de servicio (Lichtheim, 1988: 42).

3. Museo de El Cairo, JE 32138/ CGC 20543. Edición: Petrie (1900:

51-53, núm. 55, lám. xv); Lange y Schäfer (1902-1925 II: 164-167);

Landgráfová (2011: 74-78, núm. 26).

Bibliografía: Stock (1949: 46-48);

Posener (1951: 170); PM V: 113;

Schenkel (1965: 112-116, núm. 81; 1976: 57-58); Fischer (1968: 82, $84 \mathrm{n}$. 371, 149, 154, 168); Lichtheim (1988: 42-46, núm. 18, lám i= lám. II.A del presente artículo); Parkinson (1991: 93); Moreno García (1997: 85 n. 296, 106; 1999a: 101 n. 126, 175-178, 181, 202, 205; 1999b: 254; 2009: 204-205); Postel (2004: $58 \mathrm{n}$. 276, 92, 106, 108, 115, 124, 127 n. 581 , 128, 215 n. 972, 294); Landgráfová (2007: 273-276, núm. 57; 2015: 180, 183); Musacchio (2008: 52).

4. En el presente artículo no se ha podido incorporar una fotografía original de la estela debido a que los derechos de publicación pertenecen actualmente a los egiptólogos L. Postel (Université Lyon) y A. Pillon (Université Paris-Sorbonne), en el marco del proyecto del IFAO: Corpus d'épigraphie thébaine, fin Vle dynastie-début XIle dynastie. Así, la edición de este artículo está basada en la reproducción de Petrie (1900: 51-53, núm. 55, lám. xv), copia muy fiel del original (lám. II.B). Al respecto, quisiera expresar mi agradecimiento al Dr. Postel por facilitarme información acerca de su proyecto.

\section{Introducción}

A inicios de la XI dinastía la unificación de Egipto de finales del III milenio a.C. todavía no había tenido lugar. Sin embargo, la representación del estatus de los primeros reyes tebanos, su rol divino y simbólico, su familia y su poder económico y político fueron las bases sobre las cuales las formas y principios de la administración provincial del Reino Medio se desarrollaron. Estos reyes no sólo se dedicaron a conquistar tierras, también tomaron una serie de medidas de reorganización del territorio en las provincias que iniciaron el cambio y modificaron el paisaje. El objetivo era crear una organización fiscal eficiente, diseñar nuevas vías de comunicación, explotar los recursos existentes y proteger las fronteras.

Estas medidas administrativas son posibles de rastrear en la documentación contemporánea. Aunque los corpora documentales más importantes de finales del III milenio proceden de Tebas y Abidos, el de Dendera ${ }^{1}$ también contiene documentos relevantes, como la estela de Rediukhnum (Petrie, 1900: 51-53, lám. $\mathrm{xv})^{2}$. Este texto aporta información valiosa sobre la reorganización administrativa de las provincias más meridionales del Alto Egipto. El mismo Rediukhnum es un oficial $(s r)$ enviado por la reina Neferukayet a Dendera en calidad de imy$r 3 p r$ a administrar una hacienda ganadera $(m \underline{d} t)$ de su propiedad. Pero es el pasaje que menciona el "envío de $h q 3 w h w w t$ y srw a todas las provincias del Alto Egipto" el que, quizás, ofrece uno de los mejores ejemplos de ordenación del territorio provincial de inicios de la XI dinastía. Así, la estela de Rediukhnum ofrece la oportunidad de estudiar las medidas de reinstauración de las instituciones tradicionales estatales anteriores a la reunificación del estado del Reino Medio, el rol y funciones que tenían estos oficiales enviados a las provincias y cómo era su relación con la realeza o la administración central.

\section{Estela de Rediukhnum. Museo de El Cairo JE 32138/CGC 20543³}

\subsection{Inscripción (lám. I)}

Lineas 1-6: Fórmula de ofrendas y titulatura

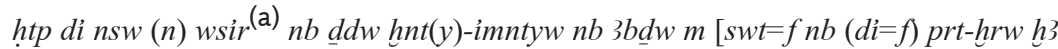

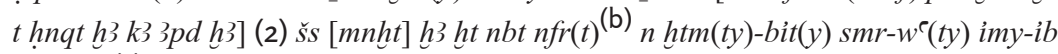
$n n b t=f^{(\mathrm{c})}$

(1) Una ofrenda que el rey da (a) Osiris, señor de Busiris, Khentimentiu, señor de Abidos, y en [todos sus lugares (para que él dé) una ofrenda invocada de miles de hogazas de pan y jarras de cerveza, miles de cabezas de ganado y aves, miles de] (2) piezas de alabastro y [lino], y miles de cosas buenas de todo tipo para el portador del sello real del Bajo Egipto, amigo único, favorito (lit. "el que está en el corazón") de su gran señora,

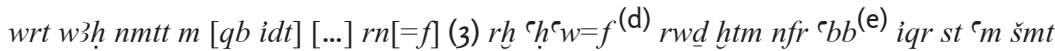

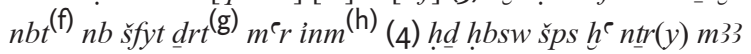


de pasos tranquilos en [el hacer una libación (?) y un donativo (?)] [...] [su] nombre, (3) el que es consciente de su rango (lit. "el que conoce su posición"), de sello firme, apuesto (lit. "bueno de forma"), de rango excelente, el que conoce toda tarea, dignificado (lit. "señor de prestigio"), generoso, educado, (4) de vestidos blancos, atractivo (lit. "noble de cuerpo"), de apariencia divina (lit. "divino de ver"),

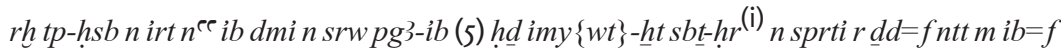

el que conoce el método correcto de actuar, piadoso (lit. "liso de corazón"), compañero de los $s r w$, generoso (lit. "abierto de corazón"), (5) sincero (lit. "blanco de pensamiento"), agradable con el solicitante que expresa su voluntad,

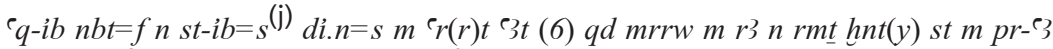
$i m y-r^{3} p r^{(\mathrm{k})} i m 3 \underline{h} w r d i-(w i)-\underline{h} n m(w)^{(\mathrm{l})} \underline{d} d=f$

íntimo de su señora, su favorito (lit. "el que pertenece a su afecto"), al que ella ha colocado en una gran hacienda, (6) distinguido, amado en boca de la gente, primero de su rango en la gran casa, el imy-r? $p r$, el imakhu, Rediukhnum, dice:

\section{Notas del texto:}

(a) Fórmula de ofrendas originalmente propuesta por Gardiner (Davies y Gardiner, 1915: 79-81), y ahora comúnmente aceptada, tras el estudio de Lapp $(1986)^{5}$. No se sabe con certeza cuándo queda establecido el cambio en la fórmula de ofrendas del Reino Medio. Sin embargo, es muy posible que se diera durante el reinado de Intef II Wahankh (Lichtheim, 1988: $44 \mathrm{n}$. 1). Unos de los primeros ejemplos se encuentran en la estela de Qemen ${ }^{6}$ con la estructura di inpw. En este caso, Anubis es el oferente del difunto.

(b) Reconstrucción realizada a partir de la estela de Megegi (Intef III) ${ }^{7}$, cuya

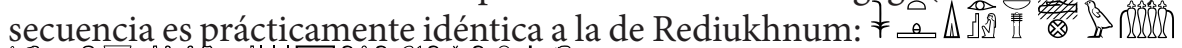

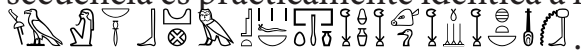

(c) Esta secuencia de títulos únicamente marca el rango del individuo. Sin embargo, resulta muy probable que en tiempos más tempranos tuvieran un significado funcional. Para este periodo, a inicios del Reino Medio, estos títulos se fosilizan y pasan a tener una connotación honorífica. El rango del individuo suele denotarse en la suma de sus títulos. Así, la gradación básica de rango de menor a mayor durante el Reino Medio es la siguiente: 1) smr-w'ty;

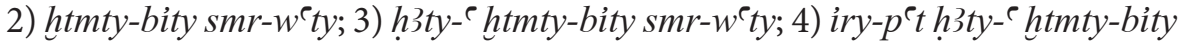
smr-wety.

(d) Durante el Reino Antiguo existía una única secuencia de títulos, con los más relevantes al principio y los menos al final (Baer, 1960). Sin embargo, la misma estela de Rediukhnum nos muestra la nueva organización que va a perdurar durante todo el Reino Medio. Los títulos ahora se presentan organizados en grupos, con aquellos que muestran el rango del individuo al principio ( $h \mathrm{tm}(\mathrm{ty})$ bity, $s m r-w^{\top}(t y)$ e imy-ib $n n b t=f$ ) y los que definen su función al final (imy-r3 pr) (Helck, 1986: 600). En el cuerpo de la secuencia se encuentran otros títulos de índole autobiográfica (Grajetzki, 2013: 216) ${ }^{9}$. Estos están divididos en tres
5. Por otro lado, Franke (2003) no está de acuerdo con esta interpretación y no cree que exista tal cambio.

6. Museo Egipcio de Turín, Inv. Cat. 1517, I. 2; Piehl (1886-1903 I: lám. IxxxiiA; II: 69-70); Lichtheim (1988: 61-62, núm. 23).

7. MMA, Inv. Cat. 14.2.6, I. 1-2; Clère y Vandier (1948: 18, núm 22); Stock (1949: fig. 17, lám. xii); Schenkel (1965: 108-109, núm. 77); Landgráfová (2007: 240, núm. 40; 2011: 20-21, núm. 8).

8. Este orden es propuesto por Willems (2013: 372-373). Sin embargo, Schnare (1981: 118), en su tesis doctoral, aprecia cómo el título htmty-bity aparece más frecuentemente en solitario que $s m r$-w'ty en las estelas del Reino Medio.

9. Con esta nueva estructura, a diferencia de lo que sucedía en el Reino Antiguo, se puede distinguir claramente el cargo o función del individuo de los títulos que muestran únicamente su rango (honoríficos, heredados, etc.). 
10. Museo Británico, EA 614, I. 1-3; Budge (1911-1922 I: lám. 49-50); Blackman (1931: 55-61, lám. viii); Clère y Vandier (1948: 15-17, núm. 20); PM I: 596; Schenkel (1965: 103-107, núm. 75); Lichtheim (1973: 90-93; 1988: 46-49, núm. 19, lám.

ii); Landgráfová (2007: 245-248, núm. 44; 2011: 10-15, núm. 5).

11. Museo de Antigüedades de Leiden, V 6/Inv. Cat. 4, I. 14; Boeser (1909: lám. iii); Lichtheim (1988: 111-113, núm. 48); Landgráfová (2007: 190-191, núm. 18; 2011: 190-193, núm. 56). 12. FCD: 41,15 . 13. Véase n. (d), I. 3. 14. Wb I: 178, 7; HL1: 136.

15. Qubbet el-Hawa 35, col. D.6-D.7; Morgan y otros (1894: 174-176); Urk. I: 131-135, núm. 27; PM V: 237; Lichtheim (1988: 15-18, núm. 3); Strudwick (2005: 333-335, núm. 242).

16. Qubbet el-Hawa 35, col. D.10. 17. Deir el-Gebrawi 67, col. 28; Davies (1902 II: 27-31, lám. xxii-xxv); Urk. I: 76-79, núm 52; PM IV: 242; Schenkel (1965: 42-44, núm. 34); Lichtheim

(1988: 23-24, núm. 6); Strudwick (2005: 366-368, núm. 269).

18. Asiut V/M11.1, col. 22; Griffith (1889: lám. 15); Brunner (1937: 64-69); PM IV: 264; Schenkel (1965: 71-74, núm. 57); Lichtheim (1988: 28-29).

19. Museo Británico, EA 614, I. 4-5. Traducción de M. Lichtheim (1988: 49 n. 1).

20. Jones (2000: 474, núm. 1765). grupos: el primero, que termina con $n \underline{t} r(y) r m 33$ "de apariencia divina", describe su apariencia física y su buena conducta; el segundo, que comienza de nuevo con $r h$ "el que conoce...", muestra su personalidad; y el último describe la relación personal que tiene con $n b t=f$ "su señora", la reina. Los tres grupos forman un apéndice a su titulatura y el discurso está en tercera persona. A partir de aquí será Rediukhnum el que hable. Al igual que en la estela de Tjetji (Intef II Wahankh/Intef III) ${ }^{10}$, esta secuencia acaba de la misma forma, con el título de oficial, el nombre y $\underline{d} d=f$.

(e) El significado de $n f r-` b b$ tiene una interpretación complicada. En la estela de Intef (Amenemhat II) ${ }^{11}$ aparece este título con los determinativos $\unlhd$. Con esta grafía ' $b b$ es traducido por Faulkner ${ }^{12}$ como "use the pitchfork", de modo que el título entero sería "bueno en el uso de la horca", traducción que encajaría con el título que le antecede, $w 3 \underline{d}$-sk3 "de cultivos afortunados". Sin embargo, esta traducción no parece apropiada para Rediukhnum porque no presenta los determinativos que muestra Intef y porque no concuerda con el resto de la secuencia ${ }^{13}$. Por lo tanto, como $~ \ b b$ también está relacionado con las cerámicas y sus diferentes formas ${ }^{14}$, puede significar "de forma buena" ("bien formado" "apuesto"). En este caso, esta traducción sería más acorde con la secuencia que describe el físico del individuo.

(f) Lichtheim (1988: 42) propone esta construcción: íqr st-` $m$ šmt nbt "efficient in performance at every task".

(g) Schenkel (1965: 112 n. f.) lo traduce como "großzügigen" y entiende que Rediukhnum se califica como alguien que ofrece frecuentemente su "mano" a los demás.

(h) Traducción de Lichtheim (1988: 42). Landgráfová (2011: 75) lo traduce como "one faultless of appearance".

(i) El recurso utilizado para definir a Rediukhnum es la enumeración de epítetos compuestos "sustantivo-sustantivo" (en genitivo directo) o "adjetivo-sustantivo" (construcción $n f r h r$ ). Entre ellos se cuentan: $r w \underline{d}$ htm "de sello firme",

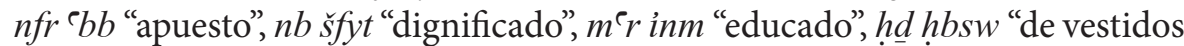
blancos", šps $h^{e}$ "atractivo", $n^{\top e} i b$ "piadoso", pg\}-ib "generoso", $h \underline{d} i m y\{w t\}-\underline{h t}$ "sincero", etc. A lo largo de la XI dinastía se aprecia un aumento de los sintagmas nominales en detrimento de las oraciones verbales. Si Pepynakht (Pepy II), en su autobiografía, dice: $m h n b(=i) i b=f i m(=i)$ "Mi señor llenó su corazón conmigo" 15 , Rediukhnum simplemente se describe como ' $q$-ib $n b t=f$ "íntimo de su señora". Sin embargo, los títulos compuestos aparecen tímidamente por primera vez en la VI dinastía. El mismo texto de Pepynakht empieza a utilizar palabras como $r s-t p$ "el que vigila" ${ }^{16}$. Es durante el Primer Periodo Intermedio que su uso empieza a ser más frecuente y se encuentran palabras como mnh-qd "amable"17 en la tumba de Henqu o 'q3-bi3t "centrado"18 en la falsa puerta de Khety I. En cualquier caso, durante la XI dinastía este recurso explota todo su potencial. Otros ejemplos se encuentran en la estela de Tjetji: $s t$ - $h r$ "cuidado", st hrt-ib "confianza"19, etc.

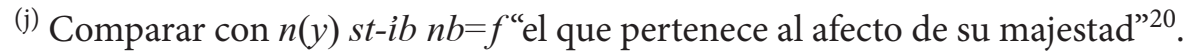


(k) La estela de Rediukhnum, a pesar de ser una de las más elaboradas y complejas de Dendera, cuenta tan solo con los títulos de rango htm(ty)$\operatorname{bit}(y)^{21}, s m r-w^{\top}(t y)^{22}$ e imy-ib $n n b t=f^{23}$, y el título funcional imy-r3 $p r^{24}$. De hecho, tanto en el arquitrabe de Hornakht ${ }^{25}$ como en la estela de Iteti ${ }^{26}$, ambos ejemplos de Dendera y de la XI dinastía, los propietarios solo muestran el título de $s m r-w^{\top} t y$. Incluso se pueden encontrar individuos que no escriben título alguno; tal es el caso de Intef, hijo de Ka, en Dra Abu el$\mathrm{Naga}^{27}$. Sin embargo, la documentación anterior a la XI dinastía es abundante en títulos: Meni (IX/X dinastía) ${ }^{28}$ porta los títulos de rango h3ty- ${ }^{29}$, $s m r-w^{\top} t(y)$ y htmty-bity, y otros títulos funcionales como $h q 3 h w t^{3 \dot{0}}, h r p m$ hb3t "director en la barca Khebat (=barca de Hathor en Dendera)"31, y hry$h b t$ "sacerdote lector"32; y Neferseshempepi/Seneni (IX/X dinastía) ${ }^{33}$ los títulos de rango htmty-bity y $s m r-w^{\top} t(y)$, y otros títulos funcionales como

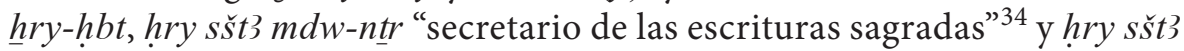
htmt nswt "secretario del sello real" 35 . A pesar de esta clara disminución de los títulos en cantidad y en importancia desde inicios de la XI dinastía, Fischer (1968: 177) observó que el número de estelas autobiográficas en Dendera aumentó. Todo ello es un reflejo de la pérdida de la centralización del gobierno menfita y el resultado de la autonomía local y privada ganada en los últimos años (Musacchio, 2008: 57).

(1) Nombre traducido como "Khnum me entregó" (se entiende: "al mundo", "a mis padres") ${ }^{36}$. Está documentado y catalogado, fundamentalmente, en el Reino Medio y, seguramente, provenga de la tebaida. Algunos ejemplos de la XII

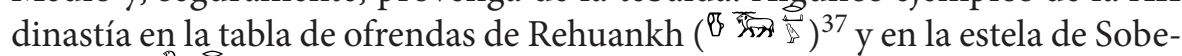
khetep $(\text { \& } 3)^{38}$.

\section{Líneas 6-10: Elogio a nbt=f"su señora"}

$\operatorname{ink}^{(\mathrm{a})} m r y n b t=f(7) \underline{h} s y=s m \underline{h} r t-\underline{h} r w n t r^{c} n b$

Soy yo el amado de su señora (7) y el que es favorecido por ella en el transcurso de cada día.

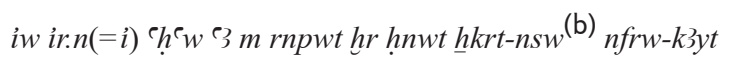

Yo he pasado un largo periodo de años bajo (el mandato) de la señora, ornamento real, Neferukayet,

wrt (8) $m k 3$ sw=s hnt(y)t $m s w t=s$ C 3 itw hntt $m w w t^{(\mathrm{c})}$ hhyt pt tn $n$ itw=s šps $(t)$ hnt $(y) t$

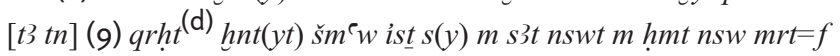

grande (8) en sus kas, primera de su rango, de padres excelsos y de madres excelentes, para cuyos antepasados se ha elevado este cielo, noble, la que está al frente de [esta tierra], (9) espíritu ancestral, la que está al frente del Alto Egipto mientras es hija real y esposa real amada de él.

$i w^{\top} \cdot n=s m w(w) t=s n b t[g] r g\left[. n=s^{(\mathrm{e})} \check{s} m^{c} w\right]^{(\mathrm{f})}(10) h 3 t(y) r m \underline{t}^{(\mathrm{g})} t p(=s) r 3 b w p h t=s r$

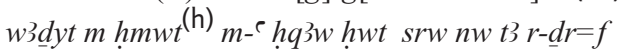

21. Jones (2000: 763, núm. 2775); Wb I: 435, 8; HL1: 626. 22. Jones (2000: 89, núm. 3268); Wb I: 278, 11; HL1: 708. 23. Jones (2000: 893, núm. 3269). 24. Jones (2000: 114, núm. 461); Wb I: 514, 10-13; HL1: 55-56. 25. Museo de El Cairo, JE 46048, I. 1; Fischer (1968: 230); Abdalla (1993: 249-253; fig. 1, lám xxiv1); Musacchio (2006: 77-85, fig. 2; 2008: 55, fig. 16). Primera referencia a este documento en: FCD: 196, 11. 26. Museo de El Cairo, JE 46049, col. 2; Abdalla (1993: 253-254, fig. 2, lám. xxiv2); Musacchio (2008: 55, fig. 17). 27. Museo Británico, EA 1203, I. 1-2; Budge (1911-1922 I: lám. 53; 1914: lám. vii); Clère y Vandier (1948: 19, núm. 23); Grdseloff (1948: 6o); Bennett (1962: 158-159); PM I: 596; Schenkel (1965: 226-227, núm. 374); Landgráfová (2007: 251-252, núm. 45; 2011: 24-27, núm. 9). Aunque no se haya hallado un ejemplo similar en Dendera es muy posible que hubiera existido.

28. Museo de El Cairo, JE 32136/ CGC 1662; Petrie (1900: lám. i); PM V: 110; Borchardt (1937-1964 II: 120-121, lám. 88); Schenkel (1965: 34-36, núm. 74); Fischer (1968: 170175); Musacchio (2008: 52, fig. 2-3). 29. Jones (2000: 496, núm. 1858); Wb III: 25, 7-8; HL1: 506-507.

30. Jones (2000: 670, núm. 2453). 31. Jones (2000: 734, núm. 2671). 32. Jones (2000: 89, núm. 3268); Wb III: 395, 4-10; HL1: 640-641. 33. Museo de El Cairo, JE 32146/ CGC 1659, col. 2-3; Petrie (1900: 47, núm. 50, lám. vii); Borchardt (19371964 II: 118); Fischer (1968: 119128); Musacchio (2008: 53, fig. 8). 34. Jones (2000: 622, núm. 2281). 35. Jones (2000: 638, núm. 2339). 36. PN I: $228,8$.

37. Museo de El Cairo, JE 39587/CGC 23045, col. D; Kamâl (1909: 39-42).

38. Museo del Louvre, $C$ 31; Rougé (1872: 90); Gayet (1889: lám. Ivii-Iviii). 
39. Museo Británico, EA 614, I. 3-4.

40. Jones (2000: 794, núm. 2899); Wb III: 401, 6-10.

41. MMA, Inv. Cat. 14.7.13, I. 5-6; Urk. I: 297-298, núm. 176A; Strudwick (2005: 118-119, núm. 32). 42. "Ubicación desconocida", I. 10; Urk. I: 302-303, núm. 176G; Strudwick (2005: 119-120, núm. 33). 43. Jones (2000: 796, núm. 2900).

44. Beni Hasan 3, I. 18-19=624; Breasted (1906: 279-289); Urk. VII: 25,17-35, 16, núm. 6 .

45. Este título, aquí utilizado para la reina, también es usado por algunos oficiales como Neheri (Hatnub 20, col. 2; Anthes, 1928: 42-47, lám. 18). 46. Wb V: 187, 16-20; HL1: 904; FCD: 291, 3-10.

47. Deir el-Gebrawi 67, col. 18-19. 48. MMA, Inv. Cat. 65.107, col. 8; Allen (1921: 55-62); Goedicke (1960: 288-291, fig. 1).

49. Enseñanzas para Merikare P106-P109=xxxviii; Helck

(1977); Lichtheim (1973: 97-109); Parkinson (1997: 212-234).
Ella ha tomado herencia de todas sus madres y ha repoblado el [Alto Egipto], (10) vanguardia del pueblo, (su) sur hasta Elefantina, su norte hasta (el nomo de) Wadjit (=Afroditópolis), con mujeres y por medio de $h q 3 w h w t$ y srw de todos los rincones de la tierra (lit. "de la tierra hasta su final").

Notas del texto:

(a) La siguiente secuencia, al igual que en la estela de Tjetji3", continúa con "Soy yo el amado de su señora ("señor" en la estela de Tjetji)", con "Yo he pasado muchos años bajo...” y termina dando una descripción de las fronteras del reino tebano.

(b) $\mathrm{O}$ "la que es ornamentada por el rey" 40 . En un principio se propuso que el título $h k r t-n s w$ fue usado por mujeres que no podían ser identificadas como doncellas o concubinas reales y que eran entregadas a oficiales distinguidos una vez su carrera en el harén real había finalizado (Kees, 1933: 77). Sin embargo, parecen ser mujeres pertenecientes a la corte provenientes de todas las clases sociales, y que podían llegar a ser esposas reales (Drenkhahn, 1976). Troy (1986: 77-79) cree que estas mujeres tenían, sobre todo, funciones musicales en los rituales relacionados con Hathor. La autora argumenta que este título está íntimamente relacionado con las $n f r w-n s w$, y concluye que únicamente se diferencian en que las $\underline{h} k r w-n s w$ son mujeres casadas. En cualquier caso, expone que un uso temprano de este título estaría vinculado a mostrar el alto rango de la mujer que lo porta. Neferukayet es un ejemplo de ello ya que, como se ve más adelante (l. 9), también es esposa real. Otro ejemplo de este uso arcaico del título se encuentra en los decretos de Coptos $\mathrm{J}^{41}$ y $\mathrm{K}^{42}$ de Neferkauhor (VIII dinastía), donde aparece su hija, Nebet II, como esposa real del nomarca de Coptos, Shemay, con el título de $\underline{h} k r t-n s w w^{\top} t t$ "único ornamento real"43.

(c) La mención a la herencia recibida de los antepasados, ya sean maternos o paternos, es un tema muy recurrente en esta época. Ver la mención a la $i w^{\top} t$ $i t=i m w t=i$ "la herencia de mi padre y de mi madre" 44 en la biografía de Khnumhetep II (Amenemhat II).

(d) Traducción de Landgráfová (2011: 76) ${ }^{45}$.

(e) El verbo grg $^{46}$ tiene diferentes acepciones como "fundar", "establecer", "poblar" u "organizar", y todas están relacionadas con el principio, establecimiento y origen de algo, o la vuelta a un estado anterior. Se ha escogido, para este caso, la acepción "repoblar" debido a un paralelo en la tumba de Henqu: $i w \operatorname{gr} g r g . n(=i)$ niwwt b3gb $\{r\} \mathrm{m} \mathrm{sp} 3 t$ tn $m(?)[n]$ w kt sp3wt wnw iw = sn n mrw $i m=s n$ ir.t $(w)=s n m s r(w)$ "Yo también repoblé las ciudades que estaban debilitadas en esta provincia con personas (?) de otras provincias. Aquellos que en ellas formaban parte de los sirvientes se convirtieron en $s r w^{\prime \prime 4}$. Otras acepciones, como "reconstruir", se documentan en la estela de Djemi (Intef II Wahankh $)^{48}$ y en las Enseñanzas para Merikare ${ }^{49}$.

(f) Lange y Schäfer (1902-1925 II: 165) reconstruyen la secuencia de este modo: [" ["habiendo tomado herencia de todas sus madres y habiendo repoblado el [Bajo y Alto Egipto]”). Sin embargo, la reconstrucción de Posener 
(1951: 170), la cual he utilizado, me parece más correcta por dos razones: en primer lugar, el pronombre sufijo en tercera persona acompañando al verbo no se omite en ningún caso en todo el texto cuando se refiere a la reina; y en segundo lugar, la mención al "Bajo Egipto", sin contar que sería la única de todo el documento, estaría descontextualizada históricamente en este documento.

(g) Este término, aquí un epíteto para el Alto Egipto, es utilizado por el mismo Ankhtifi de Moalla para su persona ${ }^{50}$.

(h) De acuerdo con Moreno García (1999a: 176-177), estas hmwt probablemente sean sirvientes, tal y como se ha visto en un pasaje similar de la biografía de $\mathrm{Henqu}^{51}$. Sin embargo, no se puede descartar que lo que se esté expresando aquí sea una política dirigida a aumentar la población del territorio. Tal es el propósito de Hornakht cuando manifiesta haber dado $\underline{h r d w t} n$ hiw "mujeres jóvenes a maridos" 52 . Una referencia más dudosa puede hallarse en la biografía de Henu (Primer Periodo Intermedio) ${ }^{53}$.

\section{Líneas 10-23: Rediukhnum en Dendera y la fórmula de Abidos}

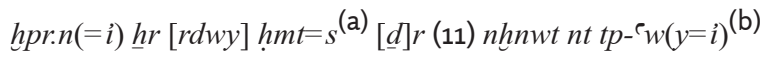

Es bajo los [pies] de su majestad (=la reina) que yo he crecido [desde] (11) la juventud de mis antepasados.

$i h r$ rh $=$ siqr st $\left.^{-}{ }^{-}=i\right)^{(\mathrm{c})} m \underline{d} d(=i) m \underline{t} n$ n srw

Por ello, ella pudo saber de la excelencia de mis actos y de mi lealtad a los srw.

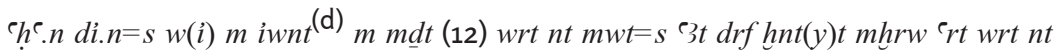
$\check{s} m^{\ulcorner} w^{(\mathrm{e})}$

Entonces, ella me instaló en Dendera, en la granja vacuna (12) grande de su madre, de inscripciones abundantes, principal negocio y gran morada del Alto Egipto.

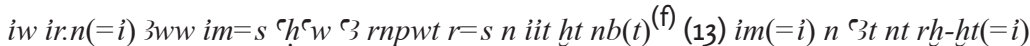

Yo pasé mucho tiempo en ella, un largo periodo de años dedicado a ello sin que ninguna cosa (mala) ocurriera (13) por mi (culpa), debido a mi gran sabiduría.

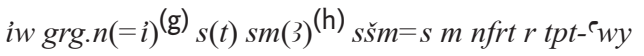

Yo lo he reorganizado en su totalidad y (ahora) su administración está mejor que antes.

$\operatorname{srw} \underline{d} . n(=i) g m t . n(=i)$ wst $\underline{t} \underline{s} . n(=i) g m t . n(=i) f d q(14) m h \cdot n(=i)^{(\mathrm{i})} g m t . n(=i) i \underline{i t} \underline{\mathrm{j}}^{(\mathrm{j})} n t h(=i)$ hbw nbw gm.n $(=i)$ ir $m$ pr ${ }^{(\mathrm{k})} p n$

Yo hice productivo lo que encontré arruinado, arreglé lo que encontré roto, (14) enriquecí lo que encontré pobre y no desatendí ninguna fiesta que yo encontré que se hacía en esta casa.
50. Moalla, tumba de Ankhtifi, col. I. .2; Vandier (1950: 171); Schenkel (1965: 45-57); Lichtheim (1988: 24-26, núm. 7); Serrano Delgado (1993: 85-89, núm. 16).

51. Véase n. (e), I. 9.

52. Museo de El Cairo, JE 46048, I. 5 . 53. Museo de El Cairo, CGC 20011, col. 7=c1; Lange y Schäfer (19021925 I: 10-11; IV: lám. i); Clère y Vandier (1948: 3, núm. 4); Landgráfová (2007: 241-242, núm. 41) 
$s \underline{t} m n(. w) h r$ imnwt $n b(t) h \underline{b} n b \operatorname{ir}(. w) r s w w=f h r-t p s n b n$ hnnwt(=i) nfrw-k3yt (15) $n$ $n h h n \underline{d} t$

Un sacrificio se realizó con todas las ofrendas diarias y toda fiesta fue hecha acorde a su día en nombre de la salud de mi señora, Neferukayet, (15) para siempre y para (toda) la eternidad.

$\operatorname{grg} . n(=i) \operatorname{prm} \operatorname{tp}-n f r^{(\mathrm{l})} \mathrm{s}^{\mathrm{C}} . n(=i) s b h t=f n b t$

Yo organicé la hacienda exitosamente y aumenté todos sus departamentos.

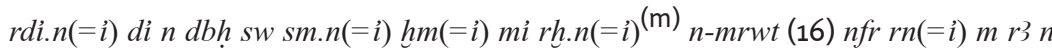
$t p(y) w-t 3$

Yo di provisiones al que las pidió, ayudé al que no conocía como si fuera conocido mío con el fin de que (16) mi nombre fuera bello en boca de (todos) los que pisan la tierra (lit. “los que están encima de la tierra”).

ink wnnt sr 3 n ib=f hnn bni $n$ mrwt

Yo soy ciertamente un $s r$ bondadoso (lit. "grande de su corazón”) y una planta dulce de deseo.

$n$ th. $n(=i) n m h \cdot n(=i) i b n$ b $3 g \cdot n(=i)[h r] \underline{h r t}-\left[{ }^{\top} w(=i)\right]^{(\mathrm{n})}$

No me emborrachaba, ni era negligente, ni era descuidado [con] mis criados.

(17) in ib $(=i)$ shnt st $(=i)$ in $q d(=i)$ rdi w3h ḩ $3 t$

(17) Fue mi voluntad la que hizo ascender mi rango, y fue mi carácter el que hizo que perdurara en primera línea.

$i r . n(=i) n n m i-q d i r . n(=i) i s \underline{t} w(i) m i m y-i b n b t=f^{(0)}$

Hice esto de acuerdo con como he actuado (siempre) siendo el favorito de su señora.

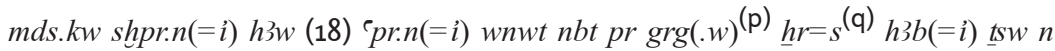
$\operatorname{gmt} . n(=i) h r$

Siendo perspicaz creé (más) propiedades, (18) equipé cada servicio dejando la hacienda organizada con ellos y envié ayuda a lo que yo encontré debilitado.

dd is iw 3h(t) wrt irr s 3h(t) nib=f(r) $n n b t=f(s) h n t t$ (19) $m n w=f^{(\mathrm{s})}$

Se dijo: "Es muy beneficioso que un hombre haga lo que considera beneficioso para su señora, la que promueve (19) su monumento".

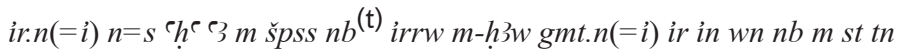


He creado para ella una gran pila de todo tipo de riquezas, las creadas (por mí), además de las que yo encontré hechas por todos mis predecesores en el cargo (lit. "los que han existido en esta posición").

sw3.n(=i) mity(=i) nb wn hr hrp m pr pn

Yo superé a todo mi igual que ha estado administrando esta casa.

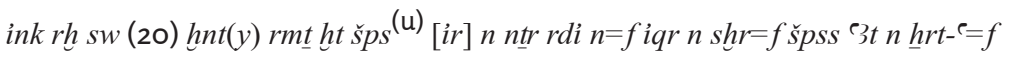

Soy yo el que se conoce a sí mismo, (20) el que está al frente de la gente, rico en madera [hecha] por el dios, uno al que se le ha dado la excelencia debido a su planificación y gran riqueza, y debido a sus acciones.

$h n w t(=i) m n b t \check{s} m^{\complement} w$ m stht $t 3 w y(m)$ wrt $n t t 3 p n$

Mi señora es la señora del Alto Egipto, la distinguida de las dos tierras y la grande de esta tierra.

w3h $k 3=s(21)$ hr st wrt ir $=s$ ḥh rnpwt $m$ `nh mi $r^{\ulcorner} \underline{d} t$

Que su ka perdure (21) sobre el gran trono y que ella viva millones de años como Re eternamente.

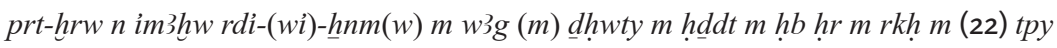
rnpt $m$ h hb wr m prt 3 t $m$ h hbw nbw mз`.t(w) $n=f$

Una ofrenda invocada para el imakhu, Rediukhnum, en (el festival de) Wag, (en) (el festival de) Djehuty, en el festival de Khedjedet, en (el festival de Horus), en el festival de Rekeh, en (22) el festival de primero de año, en el gran festival, en (el festival de) la gran procesión y en todos los festivales.

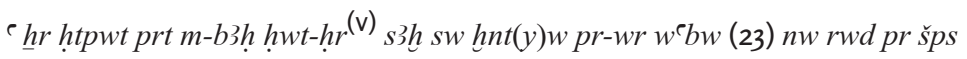

Que una mano de ofrendas que han salido al frente de Hathor sea extendida hacia él y que lo transfiguren los primeros de la gran casa y los sacerdotes-wab (23) de la terraza de la noble casa (=templo).

$w p=f w 3 w t m r r t=f m$ htp $s p 2$

¡Que él abra los caminos que desea en paz! iEn paz!

ims $h \underline{w} w$ rdi-w(i)- $\underline{h} n m(w) \underline{d} d(=f)$

El imakhu, Rediukhnum, dice:

ink ir qd=f mrrw rmt $m \underline{h}$ hrt-hrw $n t r^{\complement} n b$

Soy yo el que hace que su reputación sea amada por la gente en el transcurso de cada día. 
54. Museo Británico, EA 101, parte central, col. 2; Budge (1911-1922 II: lám. 1-2); Blackman (1935: 1-9, lám. i); PM V: 96; Lichtheim (1988: 122-124, núm. 56); Landgráfová (2007: 203-205, núm. 25; 2011: 216-218, núm. 65). 55. Museo Británico, EA 574, I. 2-3; Budge (1911-1922 I: lám. 8-9); PM I: 596; Lichtheim (1988: 96-98, núm. 41); Landgráfová (2007: 184-185, núm. 16; 2011: 182-185, núm. 53).

56. Museo de El Cairo, TR 3.6.25.1, I. 3; Daressy (1907: 245); Clère y Vandier (1948: 44, núm. 30); Schenkel (1965: 239, núm. 381); Landgráfová (2011: 59, núm. 19). 57. MMA, Inv. Cat. 57.95, I. 1-col. 2; Fischer (1959: Fig. 1; 1960: 258-268, fig. 1-2, lám. vii); Schenkel (1965: 236-238, núm. 380); Lichtheim (1988; 49-51, núm. 20); Landgráfová (2007: 255-256, núm. 49; 2011: 28-31, núm. 10). 58. Museo de El Cairo, JE 45058,

I. 4; Gardiner (1917: 35-38, lám.

ix); Schenkel (1965: 281, núm. 477); PM I: 617; Landgráfová (2011: 56-59, núm. 18). 59. Museo Británico, EA 614, I. 4-14. 6o. FCD: 10,7 .

61. Moreno García (1996: 116), a raíz del verbo $g r g$, traduce el término grgt como "fundación” o "establecimiento".

62. Moalla, tumba de Ankhtifi, col. I. .2.

63. Asiut III/N12.1, col. 35; Griffith (1889: lám. xi-xii);

Brunner (1937: 42-51); PM IV: 263; Schenkel (1965: 74-82).

64. Hatnub 24, col. 1; Anthes (1928: 54-56, lám. 24).

65. Hatnub 25, col. 10; Anthes (1928: 56-59, lám. 16).
Notas del texto:

(a) Reconstrucción realizada a partir de la estela de Nebipusenusret (Senusret

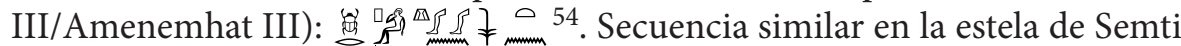
(Amenemhat II): iw di.n wi $h m=f r r d w y=f m n h n w t=i$ "Su majestad me colocó a sus pies en mi juventud" 55 .

(b) Lichtheim (1988: 43) y Landgráfová (2007: 274; 2011: 76) traducen esta frase de la siguiente manera: "I grew up under [the feet] of her majesty [since] my earliest youth", restituyendo los sufijos de primera persona y dando al genitivo indirecto final un valor adjetival. Por su parte, Schenkel (1965: 113) traduce: "Es geschah unter der Leitung ihrer Majestät, daß die Nachkommenschaft der Vorfahren groß wurde", considerando que se trata de una estructura hpr.n + circunstante + completiva de sujeto. Mi propuesta de traducción restituye igualmente los sufijos pero hace de hpr.n un perfecto enfático y mantiene el genitivo indirecto final con su sentido literal.

(c) Traducción de Lichtheim (1988: 43).

(d) La movilidad de oficiales fue algo habitual en la nueva administración de los reyes tebanos. Intefnakht (Mentuhetep III) ${ }^{56}$ fue trasladado desde Heracleópolis; Intef, hijo de Tjefi (Mentuhetep II) ${ }^{57}$, fue destinado a Heracleópolis; y Khety (Mentuhetep II $)^{58}$ menciona cierta movilidad, aunque de forma más confusa.

(e) Tanto en este texto como en el de Tjetji ${ }^{59}$ el tema principal es la relación del oficial con su señor o señora. Este se gana la confianza de su señor o señora debido a su gran lealtad, su alto prestigio y su gran competencia (Lichtheim, 1988: 49 n. 1).

(f) Oración de predicado adjetival con un infinitivo sustantivado como sujeto. El $w$ del verbo tiene un uso idiomático ${ }^{60}$. Landgráfová $(2007: 274 ; 2011: 76)$ lo traduce como una oración subordinada completiva de predicado verbal de subjuntivo y restituye un $s p$ en la oración principal negativa: $n<s p>i i h t n b(t)$ "No < ha sucedido $>$ que toda cosa (mala) haya venido".

(g) La buena gobernación (en este caso de una hacienda ganadera) se explica, durante el Primer Periodo Intermedio, a través del restablecimiento y mantenimiento del orden, reconstrucción de monumentos y abastecimiento de medios de subsistencia entre la población (Vandier, 1950: 165 n. c.), y esto se expresa mediante el verbo grg "restablecer el orden" ${ }^{1}$. Los signos $\square, ~ \sqsubset \mathrm{y} \rightleftharpoons$ representan un espacio cuyos contornos están bien delimitados y el signo $\uparrow$ representa una herramienta de trabajo. La combinación de ambos signos $(\mathbb{R}$ ) puede hacer referencia a la utilización de un instrumento sobre un terreno determinado, y, por consiguiente, al trabajo realizado en el mismo o su propia fundación o refundación. Esta acepción es recurrente en los textos del Primer Periodo Intermedio. En este caso se está haciendo referencia a la reorganización del sistema administrativo provincial del reino tebano. Ankhtifi también lo usa, pero en el contexto de sus conquistas militares: $i[w]$ in. $n w(i) h r r w t s t-h r$ $n$ ` $n \underline{h} w d \underline{d}(w) s(n b) r g r g=s$ "Horus me trajo (al nomo de) Utjesethor (=Edfu) vida, prosperidad y salud para restablecerlo"62. Otros ejemplos se documentan en las inscripciones de la IX/X dinastía de Itibi ${ }^{63}, \mathrm{Kai}^{64}$ y Neheri ${ }^{65}$. 
(h) El signo I no es del todo legible y es interpretado tradicionalmente como un ${ }^{166}$. En este caso la frase se construiría así: iw $\operatorname{grg} . n(=i) s(t) s n f r(=i) s \check{s} m=s$ "Yo lo he organizado y he mejorado su administración". Sin embargo, esta secuencia no encaja gramaticalmente con el resto de la frase ( $m$ nfrt $r$ tpt- ${ }^{\top}$ wy “está mejor que antes"). Con la sustitución de $n f r$ por $s m 3$ con valor adverbial ${ }^{67}$ se resuelve esta discrepancia.

(i) Landgráfová (2011: 76), en lugar del verbo

(j) Con la caída del Reino Antiguo y las divisiones políticas del Primer Periodo Intermedio se reforzaron los valores locales y aparecieron nuevos topos literarios como el del "administrador provincial eficaz que multiplica las riquezas que dejaron sus predecesores y reconstruye los monumentos en ruinas" (Moreno García, 1997: 59 ${ }^{68}$. Sin embargo, en la estela de Rediukhnum este topos se ve ligeramente atenuado con el del "servicio al soberano", uno nuevo que se empieza a introducir y que será común en el Reino Medio (Moreno García, 1997: 64).

(k) Con este término se refiere al centro criadero de ganado $(m \underline{d} t)(1.11)$, el cual debió ser un centro de dimensiones considerables porque también se define como 'rrt (1. 5) (Moreno García, 1999a: 177-178). Tanto pr como 'rrt están relacionados, tal y como se ve en los Textos de las pirámides: $i w{ }^{\complement} w=s n n \check{s}$ s $w 3 t$ $p r w=s n n s n s n{ }^{C} r r w t=s n n h^{\top} p(i)$ "Sus herederos están condenados a la miseria, sus casas a la destrucción y sus haciendas a la inundación" ${ }^{69}$. El término $p r$ como circunscripción territorial adquiere importancia en la IV dinastía. El texto que mejor lo corrobora es la biografía de Metjen (Esnefru). Este oficial adquiere el rol de $h q 3 p^{70}$ así como el de $h q 3 h w t^{9} 3 t^{71}$ y $h q 3 h w t^{72}$. También adopta varios títulos para una misma circunscripción, entre ellas el $p r$ Wrsh $(h q 3 h w t \text { ? } 3 t p r \text { wrsh y } h q 3 p r w r s h)^{73}$. El $p r$, como circunscripción, acabará siendo sustituido por la $h w t{ }^{\top} 3 t$ y no volverá a ser utilizado hasta finales del Reino Antiguo. Este cambio pudo deberse a un intento de Esnefru de homogeneizar la administración (Moreno García, 2007: 319; 2013: 97).

(1) Postel (2004: 128) lo traduce como "la bonne façon".

(m) Construcción idéntica en la estela de Djari (XI dinastía) ${ }^{74}$ y, con un orden diferente, en la recientemente publicada estela de Kauhmi (XI dinastía $)^{75}$.

(n) Schenkel (1965: 114), lo traduce como "meinem handeln", Lichtheim (1988: 43), como "my task” y Landgráfová (2011: 77) como "my performance”.

(o) Comparar con la estela de Intef, hijo de $\mathrm{Myt}^{76}$.

(p) El signo U17 presenta ciertas peculiaridades en este documento. Fischer (1968: 149), a pesar de que encontró la superficie de la estela seriamente dañada, pudo distinguir al menos 3 variantes: $1, \Lambda, \bigwedge(1.13,15,18)$. Las

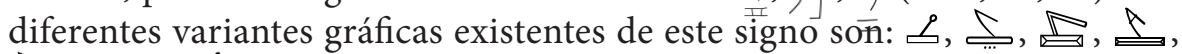
䢗, $\mathbb{\mathbb { R }}$ y $1^{77}$

(q) Lichtheim (1988: 43) y Landgráfová (2007: 275; 2011: 77) proponen otra traducción de la frase: ' $p r . n(=i)$ wnwt $n b t \operatorname{grg}(w) p r \underline{h r}=s$ "I equipped all the
66. Landgráfová en 2007 (274) da esta nueva propuesta, pero en 2011 (76) reconsidera la postura tradicional.

67. HL1: 701.

68. Otras referencias en biografías de oficiales: "desconocido" (Primer Periodo Intermedio) (Museo de El Cairo, CGC 20503; Lange y Schäfer, 1902-1925 II: 93-94); Intef, hijo de Myt (Mentuhetep II) (Museo Egipcio de Berlín, Inv. Cat. 13272; Lange, 1896: 25-35, lám. ii; Roeder, 1913: 154-155; Clère y Vandier, 1948: 44-45, núm. 31; Schenkel, 1965: 230-231, núm. 377; Landgráfová, 2007: 253-254, núm. 48; 2011: 32-35, núm. 11); Idi (VIII dinastía) (Kom el-Koffar, tumba de Shemay; Mostafa, 1984-1985: 419-429, lám. i-ii; 1987: 169-184, lám. i-ii); Khety II (IX/X dinastía) (Asiut IV/N12.2; Griffith, 1889: lám. 13-14; Brunner, 1937: 52-63; PM IV: 263); y Sarenput I (Senusret I) (Elefantina, Santuario de Heqaib 2; Habachi, 1985 I: 28-31, fig. 3a-4e; II: lám. 14-16). También en las inscripciones reales de Intef II Wahankh (Museo de El Cairo, JE 2101/CGC 20512; Lange y Schäfer, 1902-1925 II: 99-103; Clère y Vandier, 1948: 10-11, núm. 16) y Senusret I (Templo de Tod; Barbotin y Clère, 1992: 1-32, lam. 1-31).

69. Pyr. 292C-dT.

70. Museo Egipcio de Berlín, Inv. Cat. 1105-1106, col. A.1, B.6, B.8, B.10, D.1, E.a, E.b, E.f. 71. Museo Egipcio de Berlín, Inv. Cat. 1105-1106, col. A.4-A.9, A.12, B.8, B.10, D.1-D.2, E.b, E.e, E.i; Urk. I: 1-7, núm. 1; PM III: 493. 72. Museo Egipcio de Berlín, Inv. Cat. 1105-1106, col. $\mathrm{A}_{3}, \mathrm{~A}_{17}, \mathrm{~B} 9, \mathrm{Ee}, \mathrm{Ei}$.

73. Museo Egipcio de Berlín, Inv. Cat. 1105-1106, col. A.1, E.b.

74. Museo de Arte e Historia de Bruselas, E 4985, I. 4; Limme (1979: 18-19, núm. 4); Clère y Vandier (1948: 14, núm. 19); Schenkel (1965: 101, núm. 73); Lichtheim (1988: 41-42, núm. 17); Landgráfová (2007: 243, núm. 42; 2011: 7, núm. 3). 75. Almacén de El-Shaikh Hamad, Inv. Cat. 325, I. 3; Madkour (2016: 217-225, lám. 38-41). 76. Museo Egipcio de Berlín, Inv. Cat. 13272, I. 11. 77. Asiut V/M11.1, col. 18; Pyr. 1394aM; Pyr. 1589bN; Pyr. 1595aP Pyr. 1595aN; Pyr. 1837aT; Moalla, tumba de Ankhtifi, col. I. .2. 
78. MMA, Inv. Cat. 57.95, I. 8.

79. Museo Nacional de Cracovia, Inv. Cat. MNK-XI-999, col. 12-13; Černý (1961: 5-9, lám. i); Schenkel (1965: 62-64, núm. 42); Jansen-Winkeln (1988); Landgráfová (2007: 236-237, núm. 39).

80. Museo Británico, EA 614, col. 3-5.

81. Hayes (1971: 477) acepta que puede ser o de Intef II Wahankh o de Intef III.

82. Museo de El Cairo, JE 41437, I. 4. Petrie (1909: 16-17, lám. ii-iii); Clère y Vandier (1948: 14, núm. 18);

Schenkel (1965: 99-100, núm. 72; 1976: 57); Lichtheim (1988: 40-41, núm. 16); Landgráfová (2007: 244, núm. 43; 2011: 8-9, núm. 4). services by which the estate was managed". En este caso, han obviado que el escriba ha errado y se han visto obligadas a cambiar de posición $p r$ y grg para poder hacer una oración de predicado verbal de perfecto pasivo. Sin embargo, la frase puede ser traducida sin ninguna modificación como una oración de predicado pseudoverbal de forma de estado no inicial con el sujeto $(\mathrm{pr})$ precediendo al verbo $(\mathrm{grg})$.

(r) Lichtheim (1988: 44 n.12) traduce 3ht $n$ ib como "deem useful". Así, en la estela de Intef, hijo de $\mathrm{Tjefi}^{78}$, $q n$ hr ib se puede traducir como "considerar poderoso". El resto de la oración sería: irrt nbt qn(.w) hr íb n $n b(=i)$ "todo lo hecho es considerado poderoso para mi señora".

(s) Expresión de "don y contradón". La recurrente muestra de lealtad de los oficiales a la corona egipcia en las autobiografías del Reino Antiguo regresa durante la XI dinastía con una versión modificada basada en la idea de "reciprocidad”. Rediukhnum colma de riquezas a su señora a cambio de que ella le construya su tumba. Esto evidencia que la nueva monarquía tuvo que convivir con la autonomía y el orgullo personal ganados durante el Primer Periodo Intermedio. Esta nueva "clase media" es una parte importante y activa de la sociedad ya entrado el Reino Medio y es la realeza la que va a tener que adaptarse a esta realidad y no al revés (Moreno García, 2014: 12; Lichtheim, 1988: 45 n.12).

(t) En general la mención a "una gran pila" de productos implica una administración eficaz (Moreno García, 1999a: 101). Ejemplo en la estela de Merer (Primer Periodo Intermedio): iw hpr.n dd.t( $w)$ it šm ${ }^{\ulcorner} w n$ niwt iw h h. $n(=i)(n)=s$

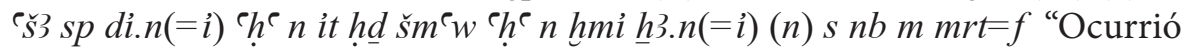
que la cebada del Alto Egipto fue entregada a la ciudad y yo medí (el grano) (para) ella muchas veces. Yo di una pila de cebada blanca del Alto Egipto y una pila de cebada-khemi y la medí (para) todos los hombres tal y como era su deseo"79.

(u) Posible construcción $n f r$ hr que el escriba ha confundido e intercambiado el orden de las palabras.

(v) Secuencia de tres deseos posteriores a la muerte, propios de la recién nacida "fórmula de Abidos". Rediukhnum se está dirigiendo a los sacerdotes de Hathor (Lichtheim, 1988: 44 n.13), denominados "sacerdotes-wab de la terraza de la noble casa (=“templo")" (Ward, 1982: 82, núm. 672). La estela de Tjetji es el documento que presenta la "fórmula de Abidos" más larga y completa ${ }^{80}$.

\subsection{Datación}

El principal problema para datar esta estela es que no menciona el nombre de ningún rey. Aunque por razones arqueológicas, paleográficas y textuales es comúnmente fechada en el reinado de Intef II Wahankh (Lichtheim, 1988: 42; Landgráfová, 2007: 273; 2011: 75; 2015: 180 ${ }^{81}$, existen otras vías de datación. Una de ellas es la mención a las fronteras del reino tebano entre Elefantina y Wadjit (nomos 1 y 10) durante la vida pública de Rediukhnum (1. 10). Estas mismas fronteras son defendidas por el rey Intef II Wahankh, tal y como aparece en la estela de Djari ${ }^{82}$. De hecho, este texto ofrece el punto exacto de la 
frontera norte en Hesy: ir.n(=i) $t 3 \check{s} r$ int ḩsy "Yo puse la frontera en el valle de Hesy" ${ }^{\text {" } 3}$. La misma frontera vuelve a ser mencionada en la estela del propio Intef II Wahankh ${ }^{84}$ en el año 50 de su reinado y último documentado. Es debido a estos paralelos que se asume que Rediukhnum y Djari fueron contemporáneos y que ambos fallecieron a finales del reinado de Intef II Wahankh. Sin embargo, en la estela de Tjetji, del reinado de Intef III, se menciona que la frontera sur es $3 b w$ pht-r $t 3-w r \underline{t} n(i)$ "Elefantina, y al norte, (el nomo de) Taur (=Abidos), (en) Tinis" ${ }^{85}$, es decir, el reino se extiende entre el nomo 1 y el nomo 8. W.C. Hayes (1971: 477) soluciona esta discrepancia proponiendo que la frontera norte en la orilla este acababa en el nomo 10 y en la orilla oeste en el 8 , de modo que, todos estos textos estarían hablando de los mismos límites fronterizos. Sin embargo, el mismo Intefiqer (Senusret I), en su descripción de las fronteras, prueba que Hayes está errado. Él dice que el nomo tinita limita al sur con iqr pht-r mn "(el nomo de) Iqer (=Dendera) y al norte con (el nomo de) Min (=Coptos)" 86 , es decir, al sur con el nomo 6 y al norte con el nomo 9. Esto quiere decir que el nomo 9 ocupaba tanto la orilla este como oeste y que las fronteras descritas por Tjetji no abarcan ni el nomo 9 ni el nomo 10. Así, la solución que aporta M. Lichtheim (1988: 45 n.14) es la de postular una incursión de duración limitada al nomo de Wadjit a finales del reinado de Intef II Wahankh, lo que explicaría por qué con Intef III las fronteras se retraen. Sin embargo, todos estos autores dan por hecho que las fronteras descritas por Tjetji son de época de Intef III, pero Tjetji encuadra esta descripción en los acontecimientos ocurridos bajo el reinado de Intef II Wahankh ${ }^{87}$, y, por lo tanto, las fronteras mencionadas seguramente sean solo del reinado de Intef II Wahankh, de un momento anterior a los hechos narrados en las estelas de Djari, Rediukhnum y el propio Intef II Wahankh. De hecho, la toma definitiva de Abidos parece tener lugar en el año 14 del reinado de Intef II Wahankh, tal y como aparece en la estela de Intef, hijo de Ka: rnpt 14 irt hadw pn rnpt $n t$ sbi $\underline{t} n(i)$ "Año 14, el hacer de esta estela, el año de la rebelión de Tinis"88. En definitiva, no existe ningún documento que pueda negar que las fronteras que mencionan Djari, Rediukhnum y el mismo Intef II Wahankh perduraran hasta el reinado de Intef III.

Otra vía de datación es el nombre de la reina Neferukayet $(1.7,14)$. A pesar de que no se ha encontrado este nombre en la documentación contemporánea ${ }^{89}$, W. Schenkel (1976: 57), M. Lichtheim (1988: 45 n.14) y L. Postel (2004: 93, 106, 124) creen que solo puede ser Neferu I, la madre de Intef II Wahankh, o Neferu II, la esposa de Intef II Wahankh y madre de Intef III, aunque no apuestan por la primera por ser demasiado lejana en el tiempo ${ }^{90}$. Sin embargo, el problema de esta propuesta es que resulta atípico que el nombre de una reina se escriba en su versión abreviada. Si se tiene en cuenta lo argumentado en el párrafo anterior y que Rediukhnum pudo haber pertenecido tanto a la época de Intef II Wahankh como a la de Intef III, resulta factible también postular que no se trata de ninguna de estas dos reinas ${ }^{91}$, sino de la esposa de Intef III, lo que haría de este documento el único en el que aparece documentado este personaje. Sin embargo, las medidas administrativas que aparecen en este texto reflejan perfectamente la reorganización administrativa que fue llevada a cabo durante el reinado de Intef II Wahankh, mientras que los textos fechados en el reinado de Intef III suelen mostrar una administración ya operativa ${ }^{92}$. No obstante, nada impide creer que Rediukhnum ya estuviera al servicio de Neferukayet en
83. Museo de El Cairo, JE 41437, col. 6. 84. Museo de El Cairo, JE 2101/CGC 20512, col. 3.

85. Museo Británico, EA 614, I. 4.

86. Museo de Antigüedades de Leiden, V 3/Inv. Cat. 3, I. 4; Boeser (1909: lám. ii); PM V: 101; Lichtheim (1988: 73-74, núm. 30).

87. iw ir.n $(=i) h^{\complement} w \Im 3$ rnpwt $h r$ hm n nb(=i) hrw3h-`nh nswt-bit(y) $s_{3}-r^{c}$ in-it $=f$ is $\underline{t} t^{3}$ pn $\underline{\text { hr }}$ st-ib=f hntt-r sbw pht-r $t_{3}$-wr $\operatorname{tn}(i)$ "Yo he pasado un largo periodo en años bajo (el mandato) de la majestad de mi señor, Horus Wahankh, señor de las dos tierras, hijo de Re Intef cuando esta tierra estaba bajo su cuidado, al sur Elefantina y al norte (el nomo de) Taur (=Abidos), (en) Tinis" (Museo Británico, EA 614, I. 3-4).

88. Museo Británico, EA 1203 , col. 15-16. De la misma época puede ser datada la estela de Djemi porque da una información similar: iw $h 3 . k(w) r 3 b d w h r(y) r s$ "Yo marché contra Abidos, que estaba en manos del enemigo" (MMA, Inv. Cat. 65.107, col. 4).

89. En un fragmento de la supuesta tumba de Intef III se lee: [...] (i) $m 3 h(y) t n f r w-k 3 w m^{c}(t)-[h r w]$ [...] “[...] La imakhyt, Neferukau, la justificada [...]" (El-Tarif, Saff el-Baqar, II 961; Schenkel, 1976: 57-58, lám. 41g, 47). Schenkel (1976: 57) dice que Neferukau es la reina Neferukayet. 90. Tanto Neferu I como Neferu II se encuentran bien documentadas: la primera se encuentra documentada en la estela de Tjetji (Museo Británico, EA 614, I. 1) y en la de Djari (Museo de El Cairo, JE 41437, I. 2-3)... (continúa en página 68)

91. Stock (1949: 47) tampoco cree que Neferu sea una abreviatura de Neferukayet. Sin embargo, él plantea que esta podría ser la reina de una hipotética dinastía de Abidos. 92. Moreno García (comunicación personal). Lo expuesto por Tjetji durante los años de reinado de Intef III es buen ejemplo de ello (Museo Británico, EA 614, I. 12-14). 
93. Véase n. (b), I. 7.

94. Ejemplo de la estela de Senendjesui (IX/X dinastía) (Museo de la Universidad de Philadelphia, E 17326; Petrie, 1900: lám ix; Fischer, 1968: 154-165, fig. 30a). Fischer (1968: 154) propone que, al no haber indicaciones de si la propiedad es oficial o privada, Senendjesui podría ser el imy-r’ $\mathrm{pr}$ de la ciudad entera de Dendera.

95. Jones (2000: 662, núm. 2426). El título $h q$ 3 se utilizaba, habitualmente, para designar al responsable de una entidad territorial de cierta importancia (Moreno García, 1996: 136-138). Algunos ejemplos en la estela de Metjen: $h q 3 \mathrm{pr}$ y $h q^{3} s p 3 t$ (Museo Egipcio de Berlín, Inv. Cat. 1105-1106, col. A.1, A.11). Un uso temprano del título imy-r3 $\mathrm{pr}$ en la Inscripción de labtet (IV dinastía) (Giza IV/G 4650; Junker, 1929-1955 I: fig. 51).

96. Inehbi (Mentuhetep II) aparece en el ataúd de la princesa Aashyt como imy-r3 pr de su hacienda privada (Museo de El Cairo, JE 47267, I. H.2; Clère y Vandier, 1948: 25-29); Intef (Mentuhetep II) en un caso similar con la princesa Kauit (Museo de El Cairo, JE 47397, col... (continúa en página 68)

97. Ejemplo de Mentuuser (Senusret I) (MMA, Inv. Cat. 12.184, I. 2; Hayes, 1953: 299-300, fig. 195; Lichtheim, 1988: 104-105, núm. 44).

98. Ejemplos en el ataúd de Buau (Mentuhetep III-Amenemhat I) (Museo de el Cairo, JE 31342/CGC 28027, I- 3.1; Lacau, 1904: 66-74) y en la estela de Tjebu (Museo de El Cairo, CGC 20005, I. 3). 99. Jones (2000: 171, núm. 652).

100. El título h3ty-` podría interpretarse como un título de rango a partir de este pasaje de la tumba de Djau (VI dinastía): iw dbh.n $(=i)$ $m-\ulcorner h m=f n \underline{d} i 3 t n t h 3 t y-\ulcorner n \underline{d} 3 w p n$ iw rdi.n $h m=f$ ir.t $t(w)$ wd $r$ dit $=f m$ h3ty-r "Yo pedí de la bondad de su majestad la designación del oficio de h3ty-` para este Djau.

(Entonces) su majestad hizo que un decreto se hiciera para que él fuera nombrado h3ty- C" (Deir

el-Gebrawi 12, col. 20-21; Davies, 1902 II: 1-13, lám. i-xiii; Urk. I: 145-147, núm 33; PM IV: 244-245).

101. La llamada "escena de las cuentas" de la tumba de Niankhkhnum y Khnumhetep (Niuserre)

muestra a 4 hombres con el título de $h q 3 w$ niwwt $t 3-m h w$ (continúa en página 68)

102. La recitación 571 de los Textos de los ataúdes (qd hwt imyt mw

“Construcción de una hwt que está en el agua") enumera las tareas más importantes del imy-r3 $\mathrm{pr}$ (continúa en página 69) época de Intef II Wahankh cuando esta todavía no era esposa de Intef III ${ }^{93}$. En definitiva, considero que Rediukhnum pudo comenzar su vida pública en Dendera durante el reinado de Intef II Wahankh y fallecer con Intef III en el trono.

\section{Contexto social y administrativo}

\section{1. "Ella me instaló en Dendera (como imy-r3 pr), en la mdt wrt de su madre, grande de inscripciones, principal negocio y gran morada del Alto Egipto” (l. 11-12).}

El imy-r3 pr era ya desde el Reino Antiguo el cabeza de la administración de las propiedades privadas (Gardiner, 1947: 46; Grajetzki, 2013: 247). Aunque existen varios textos de personajes con este título que no mencionan al propietario de la hacienda en la que el imy-r3 pr está adscrito ${ }^{94}$, lo más probable es que estas propiedades pertenecieran a altos funcionarios, incluyendo gobernadores locales. Con los cambios del sistema administrativo de la IV dinastía, el imy-r3 $p r$ acaba reemplazando al $h q{ }^{3{ }^{95}}$ y empieza a estar muy bien documentado en muchas mastabas del Reino Antiguo como el principal administrador de las haciendas de los oficiales de alto rango (Helck, 1975: 1120). En la XI dinastía, tal y como observa W. Grajetzki (2013: 247), el imy-r3 pr se convierte también en el administrador de las propiedades de la familia real ${ }^{96}$. Por lo tanto, el $i m y-r 3 p r$ estaría adquiriendo un rol más importante que en épocas anteriores (Fischer, 1968: 177). Estos oficiales, de rango inferior al de los grandes potentados locales, estaban en un segundo plano en la documentación del Reino Antiguo. Sin embargo, gracias a la "regionalización del poder" del Primer Periodo Intermedio, aparece de forma más frecuente mostrando su estatus y sus valores sociales (Moreno García, 1997: 70; 2013: 139, 147). De igual modo, los cambios ocurridos durante este periodo perdurarán en la XII dinastía y este título y su importancia no desaparecerá ${ }^{97}$. Los propios títulos de rango de Rediukhnum corroboran la importancia de este nuevo rol adquirido. Posee los mismos títulos de rango (htmty-bity, smr-w`ty) que otros imyw-3 pr de la misma época ${ }^{98}$. Es cierto que con la caída del Reino Antiguo el valor social de estos títulos de rango varía de una provincia a otra, pero en Dendera parece que este valor no cambia desde la VI dinastía, de modo que solo los cargos excepcionalmente importantes como el imy-r3 hmw-ntr "supervisor de sacerdotes"99 añadirían el título de rango h33ty-` a los ya mencionados (Fischer, 1968: 72) ${ }^{100}$. Sin embargo, J.P. Allen (2003: 16) recuerda que, en muchas ocasiones, estos títulos no representan la importancia del individuo y que se debe acudir al mismo texto autobiográfico para hacer una mejor autopsia de sus funciones. En este caso, el mismo texto de Rediukhnum muestra que él, como administrador de esta gran hacienda, una de las muchas propiedades que debió tener la reina, tuvo que ser un individuo poderoso y de gran autoridad en la provincia de Dendera (Moreno García, 1999a: 178).

¿Cuáles son las funciones reales del imy-r3 pr? Estas aparecen muy bien definidas en la tumba de Niankhkhnum y Khnumhetep ${ }^{101}$ y en los Textos de los ataúdes ${ }^{102}$. Según estos textos, las tareas del imy-r3 pr consisten en gestionar la contabilidad principal de la hacienda ( $p r$ ) (Swington, 2012: 124), aumentar sus 
riquezas y asegurar la provisión de productos (Moreno García, 1999a: 202). Sin duda, estas funciones coinciden con las de Rediukhnum, aunque, de su autobiografía se pueden extraer otras como el mantenimiento de las fiestas y las ofrendas (1. 14), y el envío de suministros al propietario de la hacienda (1. 19). En definitiva, el $i m y-r 3 p r$ en este contexto de inicios del reino tebano es el trabajador más importante y último responsable de la administración de la hacienda.

El imy-r3 pr podía estar a la cabeza de varios tipos de hacienda pero la que administra Rediukhnum es una de ganado vacuno $(m \underline{d} t)$. La producción de ganado no solo era una fuente de prestigio; también jugaba un importante rol económico. Se usaba para transporte y fuerza animal, alimento y producción de materiales (pieles, grasa, etc.). Por lo tanto, los propietarios de estas haciendas eran personajes poderosos, de un alto estatus y con un gran poder adquisitivo (Swington, 2012: 29). Pero ¿cuáles son las razones que llevaron a crear este tipo de haciendas en las provincias? J.C. Moreno García (1999b: 241-242, 256) cree que, en la VI dinastía, en base a textos como el de Ibi de Deir el-Gebrawi (Pepy II) ${ }^{103}$, la corona fomentó la creación de haciendas de producción con el objetivo de garantizar avituallamiento a las expediciones reales y explotar sus recursos; y que, las haciendas ganaderas, en concreto, jugaron un papel muy importante. El mismo Ibi admite estar $m \mathrm{~h} \mathrm{~m} \mathrm{kJw} \mathrm{m}$ ' $n h(w) t m$ '3(w) "lleno de bueyes, de cabras y de burros" 104 y la representación iconográfica de actividades ganaderas en las provincias del Alto Egipto empieza a ser cada vez más frecuente (Swington, 2012: 31, 37). Esta tradición ganadera en las provincias perduró a finales del Reino Antiguo y el Primer Periodo Intermedio, a pesar de que el rey dejó de ser una figura relacionada con ellas ${ }^{105}$. Sin embargo, durante la XI dinastía, las fuentes muestran un aumento de las referencias a las actividades ganaderas y, de nuevo, en relación con la realeza ${ }^{106}$. Es plausible creer que estas nuevas fundaciones estuvieran relacionadas con las conquistas tebanas y el aumento de tierras. Por lo tanto, la fundación de este tipo de haciendas pudo haber tenido el objetivo de abastecer a las tropas enmarcadas en este contexto de guerra civil. Quizás así se pueda explicar por qué el oficial Ip (Mentuhetep II) posee el título de imy$r 3 \breve{s} n-t 3 n b^{107}$ "supervisor de los pastos"108 a la vez que el de $i m y-r 3 m \check{s}^{c}$ "comandante" 109 . De igual modo, también pudo existir un interés por parte de la realeza en explotar estas nuevas tierras y ganar poder adquisitivo. Agentes reales como Henenu proporcionaron ganado a las provincias ${ }^{110}$ para que estas tierras se explotaran y pudieran tributar al rey ${ }^{111}$.

\section{2. “(...) ha repoblado el [Alto Egipto], vanguardia del pueblo, (su) sur hasta Elefantina, su norte hasta (el nomo de) Wadjit (=Afroditópolis), con mujeres y por medio de $h q 3 w$ hwt y srw de todos los rincones de la tierra" (l. 9-10).}

Este pasaje menciona claramente una de las medidas administrativas que se dieron a inicios de la XI dinastía. Se envían a las provinias representantes del rey, ya sean altos dignatarios del gobierno central enviados periódicamente a las provincias $(s r w)$ o administradores de las $h w t(h q 3 w h w t)$ (Moreno García, 1999a: 177). La participación del estado en la reorganización del territorio en este periodo se encuentra, tanto en los documentos del reino
103. sk w(i) $m h q$ ’ h hwt nt $p r$-šn $n t$ $3 h t$ st $\underline{t}$ t $203 \mathrm{rdi}[\mathrm{t} n(=i) n] \mathrm{hm} n \mathrm{nb}=\mathbf{i} \mathrm{r}$ $\operatorname{sinht}(=i)$ "cuando yo era $h q 3$ hwt de la hacienda de un terreno de 203 aruras que la majestad de mi señor me dio para enriquecerme" (Deir el-Gebrawi 8, I. b.2; Davies, 1902 I: 1-23, lám. vii, xxiii; Urk. I: 142-145, núm 82; PM IV: 243-244; Strudwick, 2005: 363-365, núm. 266).

104. Deir el-Gebrawi 8, I. b.2.

105. Las fuentes evocan la buena gestión eficaz del buen administrador. Ejemplos en la tumba de Henqu: iw $g r m h . n(=i) w d b w=s m$ mnmnt mhrw $=s{ }^{\top}$ 'wt "También llené sus tierras ribereñas con cabezas de ganado y sus tierras bajas con ganado menor" (Deir el-Gebrawi 67, col. 12-13); y en la estela de Heqaib (Primer Periodo Intermedio): iw ip. $n h q$ 3 $i h w(=i)$ $g m=f h 3 w(=i) h r$ ipt "El $h q$ 3 contó mi ganado y encontró que mi riqueza estaba en orden" (Museo Británico, EA 1671, col. 10; Polotsky, 1930: 194-199, lám. xxix; Landgráfová, 2007: 283-284, núm. 62).

106. Nakhty posee el cargo de imy$r 3$ $b$ nb "Supervisor de todas las cabezas de ganado" (Chatsworth, I. 1; Müller, 1933: 165-206) y Henenu (Mentuhetep II) el de imy-r 3 $b$ "Supervisor de las cabezas de ganado"...

(continúa en página 69)

107. Jones (2000: 250, núm. 903). 108. El-Saff, tumba de Ip, pared de la izquierda, col. 5, 7; Fischer (1996). 109. Jones (2000: 142, núm. 551). 110. $n h b . n(=i) s p 3 w t \check{s} m^{`} w m k 3 w$ ['wt ${ }^{3} 3$ w ] [...] "Yo abastecí las provincias del Alto Egipto con bueyes, [cabras, burros,] [...]" (MMA, Inv. Cat. 26.3.217, I. 4).

111. $b 3 k . n(=i) n=f t 3-w r$ tn $n(i) w 3$ dit pht "Yo recaudé tributo para él (=el rey) (desde) (el nomo de) Taur (=Abidos), (en) Tinis (hasta) (el nomo de) Wadjit (=Afroditópolis) al norte" (MMA, Inv. Cat. 26.3.217, I. 3). 
112. Itibi de Asiut se vanagloria de haber asegurado la frontera heracleopolitana con el reino tebano por medio de htmw "fortalezas" (continúa en página 69)

113. Al igual que los reyes de Heracleópolis, los reyes tebanos también modificaron el paisaje por medio de fortalezas. (continúa en página 69) 114. Instrucciones del Visir R25; Boorn (1988: 234).

115. En la recitación 571 de los Textos de los ataúdes ( $q$ d hwt imyt $m w$ “Construcción de una hịt que está en el agua”) se aprecia muy bien esta red (continúa en página 69)

116. ' $h^{\complement} . n$ h3b.n imy-r’ pr smsw rnsi s3 mrw n hq3 hwt n sht-m3ti hr irt `qw n hmt shtii “Entonces el imy-r3 pr más viejo, Renesi hijo de Meru, envió un mensajero al $h q 3$ hwt del campo de Mati para dar provisiones a la mujer del

campesino" (Campesino elocuente

B1 117-B1 118; Parkinson, 1991).

117. El término hwtyw es muy poco frecuente. Un paralelo en la estela de Intef, hijo de Tjefi (MMA, Inv. Cat. 57.95, I. 1-col. 4). 118. 'Iw $\left[\right.$ rdi.n $(=i)$ it $\left.\breve{s}^{\top}{ }^{\top} w\right] n$ hwtyw mi $q d=s m \operatorname{ir}(y) \Upsilon^{\complement} q w n$ hrw $n b$ "[Yo di cebada del Alto Egipto] a los trabajadores de la $h w t$ en su totalidad como encargado de las provisiones de todos los días" (Museo Nacional de Escocia, Inv. Cat. 1910.96, col. B.3-B.4; Fischer, 1968: 195, 209-213, fig. 43, lám. xxvi; Lichtheim, 1988: 33-34, núm. 12). 119. $h 3 b w(i) h m=f h r h 3 t m s ̌ w ~ p n ~(i)$

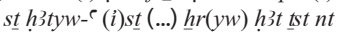
$\check{s} m{ }^{\ulcorner} w t 3-m h w$ hwwt niwwt hq3t=sn

"Su majestad me envió a la cabeza de estos soldados, además de los h3tyw- , además de (...) que están

a la cabeza de la tropa del Alto y

Bajo Egipto, de las hwwt y de las ciudades que ellos gobiernan"

(Museo de El Cairo, JE 6304/CGC 1435, col. 17-19; Mariette, 1869-188o II: lám. 44-45; Borchardt, 19371964 I: lám. 29-30; Urk. I: 98-110, núm. 67; Lichtheim, 1973: 18-23; Strudwick, 2005: 352-357, núm. 256).

120. Museo de El Cairo, JE 41437, I. 3.

121. Enseñanzas para Merikare $\mathrm{P} 62-\mathrm{P} 64=x x$ iii.

122. Instrucciones del Visir R25-R26.

123. El-Tarif, Capilla de Intef II Wahankh, II 2224, I. 5; Schenkel (1973: 215-219, fig. 1, lám. Ixxii; 1976: 50-52, lám. 42). heracleopolitano ${ }^{112}$, como del reino tebano ${ }^{113}$. Sin embargo, aunque no sea un texto coetáneo, una referencia de las Instrucciones del visir ilustra perfectamente esta modificación del paisaje provincial por parte del gobierno central: $n t f$

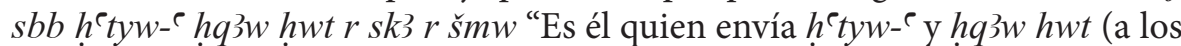
$w$ "distritos") para cultivar y cosechar (?)"114.

\subsection{1. $h q 3 w h w t$}

El $h q 3 h w t$ es el máximo responsable de la $h w t$, un centro administrativo con personal propio que asegura a la corona el control directo de una circunscripción y sus recursos (Moreno García, 1999a: 193). La $h w t$, repartida por todas las provincias, era la base del sistema tributario durante la VI dinastía. Mediante una compleja red, la $h w t$ actuaba de intermediario entre la corona y las diferentes circunscripciones, recolectaba los impuestos y los enviaba a palacio (Moreno García, 2013: 129) ${ }^{115}$. Por supuesto, para esta tarea, la $h w t$ necesitaba actuar de centro de almacenaje. En el Campesino elocuente se ve cómo se entregaban provisiones a los campesinos ${ }^{116}$, y en la estela de Seneni (IX/X dinastía) a los propios $h w t y w^{117}$ "trabajadores de la $h w t^{\prime \prime 118}$. También se encargaba de abastecer a las expediciones militares (Moreno García, 1999a: 206). Pero su participación en estas campañas no se limitó únicamente a las tareas de almacenaje. La documentación muestra que el $h q 3 h w t$ tuvo un papel activo en estas empresas. Un ejemplo magnífico para la VI dinastía lo ofrece Uni de Abidos ${ }^{119}$. Sin embargo, durante la misma época de Rediukhnum, también se encuentra esta participación directa. Djari, cuyo único cargo es el de $h q 3 h w t$, dice: ‘ $h 3 . n(=i) h n^{\complement} \mathrm{pr} h(t) y m$ imntt tny "Yo he luchado contra la casa de Khety (=Heracleópolis) en el occidente de Tinis" ${ }^{20}$. De hecho, la $h w t$ pudo haber actuado directamente como una fortaleza defensiva. En la documentación los términos hwt y mnnw "fortaleza" se suelen intercambiar. En las Enseñanzas para Merikare ambos términos se citan en un contexto de protección de fronteras ${ }^{121}$, y en las Instrucciones del visir el visir exige ser informado tanto por los hq3w hwt como por las mnnw šm`w "fortalezas del Alto Egipto" ${ }^{22}$. De igual modo, la $h w t$ también se relaciona con la fortaleza $h n r t$, la institución que, de hecho, acabará sustituyendo a la $h w t$ en el Reino Medio (Moreno García, 1999a: 182). También a principios de la XI dinastía se encuentra la relación $h w t$-fortaleza en la documentación. En un fragmento del rey Intef II Wahankh encontrado en El-Tarif, en un contexto de guerra civil entre Tebas y Heracleópolis, el texto menciona los términos hwt e ith "fortaleza" a una escala similar: ' $n h . n(=i) h q 3 w=s$ imyw-r了 $m \check{s}^{\ulcorner}=s[m h w w t] n b w$ $r s y(w) m h w w t[\ldots] m h t(y w) m[\ldots]$ ith $(w)=s$ "Yo alimenté a sus jefes y a sus comandantes [en] todas [las $h w w t]$ meridionales, en las $h w w t[\ldots]$ septentrionales y en [...] sus fortalezas" ${ }^{123}$. Este sistema de red de fortalezas no se abandonará y seguirá estando en uso en las conquistas militares de Siria-Palestina en el Reino Nuevo (Redford, 1990: 40-63; 1992: 209-213).

En definitiva, la hwt es una organización administrativa destinada a asegurar el control de la corona sobre todo Egipto y a poner a su disposición los recursos humanos y materiales del país. Es una circunscripción creada directamente por la corona y dependiente de ella con funciones administrativas, de almacenaje, de redistribución de recursos y de protección militar. No solo es centro de producción y abastecimiento, sino que también protege las fronteras del país (Moreno García, 1999a: 181, 208, 250; 2007: 325). 
El título $h q^{3} h h^{124}$ empieza a aparecer en las fuentes de la III dinastía en personajes con un rango modesto y con pocas posibilidades de ascenso social en la corte (Moreno García, 1999a: 231), aunque en la IV dinastía se ve tímidamente en oficiales de más alto rango como Metjen ${ }^{125}$. Más tarde, a finales de la V dinastía y, sobre todo, durante la VI dinastía, coincidiendo con un periodo de cambio del modelo administrativo y un intento de homogeneización del país, los $h q 3 w h w t$, al igual que las $h w w t$, aumentan, aunque de forma mucho menos homogénea en el Alto Egipto (sujeto a las peculiaridades locales, la influencia de los templos en la zona y al interés económico, político o estratégico) (Moreno García, 1997: 71; 2007: 324; 2013: 125) ${ }^{126}$. Sin embargo, en la XI dinastía y épocas posteriores este título disminuye progresivamente ${ }^{127}$ hasta prácticamente desaparecer en el Reino Medio. Tal y como se ve en la biografía de Rediukhnum, los nuevos faraones intentaron reinstaurar el sistema administrativo y productivo de la $h w t$, pero esta acabó siendo sustituida por la $h n r t$ (Moreno García, 1996: 129; 1999a: 267; 2007: 328; 2016: 506).

El $h q 3$ h $h$ t es el principal responsable de las circunscripciones estatales. Esta es la conclusión que se alcanza una vez analizados los textos de $\operatorname{Ibi}^{128} \mathrm{y}$ Metjen ${ }^{129}$. Tanto Ibi como Metjen son $h q 3 w h w t$ y los dos mencionan en sus respectivas biografías sus bienes personales, en este caso sus recompensas, pero nunca mencionan la $h w t$ propiamente dicha o los bienes producidos por la misma, lo que demuestra que esta pertenecería al estado. Los terrenos que mencionan estos dos oficiales podrían ser pagos que el rey da a cambio de la gobernancia de la $h w t$, pero nunca puede serlo la propia $h w t$. Al contrario que los imyw-r3 $p r$, el poder de los $h q 3 w h w t$ emana directamente del estado. La $h w t$, la monarquía y la organización administrativa de una región son conceptos inseparables (Moreno García, 1999a: 171, 178, 217). Sin embargo, durante el Primer Periodo Intermedio, con la realeza lejos de tener el poder que había tenido años antes, este título se siguió utilizando, y en muchos casos, controlado por familias locales (Moreno García, 1999a: 268-269; 2007: 328). A pesar de que el reino tebano era ya un estado fuerte a inicios de la XI dinastía, esta norma parece no haber desaparecido por completo. Por ejemplo, el texto de Rediukhnum muestra a la reina Neferukayet, sin hacer ninguna mención al rey, con el poder de designar este título a voluntad. Es decir, al igual que en el Primer Periodo Intermedio, una persona que no es el rey cuenta con esta potestad. Resulta factible pensar, pues, después de lo expuesto hasta aquí, que desde los primeros síntomas de crisis del reino menfita el valor y las funciones del título $h q 3$ h $h w t$ pudieron haber sido modificados. H.G. Fischer (1968: 177) observa en la documentación que, a pesar de que la institución de la ḩwt se hace más compleja después de la VI dinastía, el título de $h q 3$ 3 $h w t$, al igual que el de imy$r 3 \mathrm{pr}$, lo ostentan individuos de rango más bajo que en tiempos anteriores ${ }^{130}$.

\subsection{2. $s r w$}

Como la condición de $s r^{131}$ es muy difícil de definir, es oportuno comenzar este apartado con un análisis comparativo entre el término $s r$ y otros dos términos con los que se le suele relacionar: nmhyw y smrw. Por ausencia de documentación de la época estudiada, esta comparación solo puede efectuarse a partir de fuentes más recientes.
124. Jones (2000: 670, núm 2453); Ward (1982: 130, núm 1114); Fischer (1997: 65, núm 1114).

125. Véase n. (k), l. 14.

126. Lista de $h q 3 w$ hwt documentados para el III milenio en: Moreno García (1999a: 270277). El 90\% de las referencias abarcan desde la VI dinastía hasta finales del III milenio.

127. Varias referencias del Primer Periodo Intermedio en los textos de: Mery (Museo de El Cairo, CGC 1592, col. 4; Borchardt, 1937-1964 II: 70, lám. 79), Pemu (Museo de El Cairo, CGC 1637, I. a; Borchardt, 1937-1964 II: 103, lám. 85), Sesheshen (Museo de El Cairo, JE 29255/ CGC 1645, I. 4; Borchardt, 19371964 II: 107), Meni (Museo de El Cairo, JE 32136/CGC 1662) y Hemur (Museo del Louvre, C 198; Gayet, 1889: lám. xlii; Brovarski, 1973).

128. Véase n. 103.

129. Museo Egipcio de Berlín, Inv. Cat. 1105-1106, col. A.14, A.15, A.17.

130. En el Primer Periodo Intermedio el $h q$ 3 $h w t$ šeneni (IX/X dinastía) tan solo posee los títulos de rango smr-w'ty y htmty-bity (Museo Nacional de Escocia, Inv. Cat. 1910.96) y el $h q^{3} h w t \underline{d j}{ }^{\top} r i$ (XI dinastía) histy-` y $s m r-w^{`} t y$ (Museo de El Cairo, JE 41437, I. 1). Sin embargo, el $h q$ 3 $h w t^{\prime} I d u$ 'I, en la VI dinastía, los tres títulos de rango (h3ty-', htmty-bity y smr$w^{`} t y$ ) (Museo de la Universidad de Philadelphia, Inv. Cat. 29-66-594/D 6705; Fischer, 1968: 93-100, lám. v).

131. $s r$ es un término muy difícil de traducir. Moret (1916: 378) lo traducía como "notable", pero también se traduce como "funcionario" o, por sus competencias jurídicas, como "magistrado" (Théodoridès, 1969: 108). Sin embargo, como sus funciones también son administrativas o ejecutivas, he decidido denominarle de aquí en adelante con el término más genérico de "oficial" o con su nombre en egipcio transliterado $(s r)$. 
132. Poema de Pentaur P175P176; Gardiner (1960; 7-27); KRI II: 3-101, núm. 3i.

133. Nauri, I. 29-30; Griffith (1927: 193-208, lám. xl-xliii); KRI I: 45-58, núm. 24. Una referencia similar en el decreto de Ramsés III en Elefantina (Elefantina, I. 3; KRI V: 343-345, núm. 138B). 134. Cuento de Sinuhé B280B281; Koch (1990).

135. Instrucciones del Visir R22.

136. Cuento de Sinuhé B281.

137. $i r . n(=i)\left\ulcorner h^{c} w n b\right.$ ir.n $(=i) h r s r t$ $m i 3 w t \underline{d} b^{\ulcorner}(w) t r p h w y(=i) n n s p[s]$ $\underline{d} r(=i) \underline{d} b^{r}(w) t h r t r(=i) d r r[d i] t(=i)$ m sr nn sp s3t $(=i) n n s p[h] n r t(=i) h t$ $n b \underline{d d t} r(=i) m-b 3 h$ srw pr.n $(=i) h r=s$

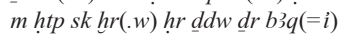
im m-b3h srw "Yo pasé todo (este) tiempo que yo pasé sobre el consejo de oficiales en la ocupación del sello hasta mi final. (continúa en página 69)

138. Museo de El Cairo, JE 6304/CGC 1435, col. 4 . 139. Museo de El Cairo, CGC 20502, col. 1; Lange y Schäfer (1902-1925 II: 93).

140. Algunas menciones de esta institución a inicios del Reino Medio en las estelas de: Intefiqer, hijo de Mentuhetepiqer: $w p \underline{h} 3 \mathrm{~m} h \mathrm{rw} n$ $s h$ "El que abre la oficina en el día de la cámara" (Museo Británico, EA 1628, I. 12); Mentuuser: ink hrp sh srw "Yo soy el administrador de la cámara de los oficiales" (MMA, Inv.

Cat. 12.184, I. 1); y Khety: ink ' $q 3$ w $m$ snkt skm-ns $m$ sh $n$ srw "Yo soy el que progresa en la oscuridad y el sabio en la cámara de los oficiales" (Museo de El Cairo, JE 45057, I. 6-7).
"1. nmhyw. En el Poema de Pentaur el rey Ramsés II se dirije a sus tropas y les dice: $i w=t n m$ nmhyw di=i iry=tn $s r w$ "Vosotros erais $n m h y w$ y he hecho que seais oficiales" 132 . Aunque del texto podemos deducir fácilmente que el $s r$ tiene un estatus más alto que el $n m h y$, definir cada título es una tarea más complicada. Posiblemente el propósito del rey en este pasaje es mostrar la fuerza de su reinado alegando que antes de él no existía un estado con un órgano de funcionarios fuerte. En ese caso, el $n m h y$, a diferencia del $s r$, podría ser una persona no integrada en el aparato del estado. El nmhy sería un "particular", "independiente", "libre", etc. y el $s r$ un "oficial" (Théodoridès, 1965: 114-118; Kruchten, 1981: 32-33).

»2. smrw. En el decreto de Seti I en Nauri se diferencia perfectamente a los

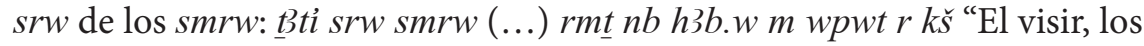
oficiales, los smrw (...) y toda la gente han sido enviados en misión a Kush" ${ }^{133}$; y en el Cuento de Sinuhé se definen mejor sus diferentes funciones: $i w=f r$ smr $m-m$ srw "Él (Sinuhé) será un $s m r$ en medio de los oficiales"134. J.C. Moreno García (1997: 101) opina que el $s r$ es un funcionario con alta responsabilidad administrativa, mientras que el $s m r$ es un cortesano sin una función específica.

En definitiva, los $s r w$ son todos aquellos oficiales que trabajan para el estado y poseen un cargo administrativo alto (Théodoridès, 1969: 108; Moreno García, 1997: 107). Tal y como se ve en las Instrucciones del visir los srw trabajan directamente para él, es decir, son trabajadores de la administración central: $n t f$ irr $n t y m$ srwt $\check{s}^{\top}{ }^{`} w$ t3- $m h w$ "Él es el que decide quien está en el cuerpo de oficiales del Alto y Bajo Egipto" ${ }^{135}$. Incluso, como se aprecia en el Cuento de Sinuhé, parece que gozan de una gran cercanía al rey: $r d i . t(w)=f m-q 3 b$ šnyt "Él (Sinuhé) será posicionado en el séquito" ${ }^{136}$. Por lo tanto, se podría decir que el $s r$ es también un funcionario de alto rango y con mucha responsabilidad. Otros textos para entender la importancia de este cargo, en este caso de la VI dinastía, son las biografías de Pepyankh y Uni. Pepyankh, de entre todos los cargos que tiene, se define como $s r$ en los asuntos de más alta importancia ${ }^{137}$, y Uni prioriza este cargo frente a todos los demás: $m h$-ib $n h m=f i m r s r w=f n b(w) r$ $s^{c} h(w)=f n b(w) r b 3 k(w)=f n b(w)$ "El hombre de confianza de su majestad (Uni) está allí más que todos sus oficiales, más que todos sus nobles y más que todos sus sirvientes" ${ }^{138}$. Sin embargo, ninguno de estos textos es de la XI dinastía. En cualquier caso, la estela de Idi, un texto coetáneo a Rediukhnum, corrobora la importancia y cercanía al rey de los srw en esta época al enumerar entre sus tareas gmt ts $m g 3 u=$ firt shr ${ }^{\complement} b 3 r 3 m$ sh $n$ srw "encontrar una solución cuando a él (el rey) se le acaba el día y expresar consejo y proveer palabras en el consejo de oficiales"139. De hecho, la mención a la $s h(n) s r w$ "cámara de los oficiales", un organismo dependiente del soberano, se da por primera vez en el Primer Periodo Intermedio (Moreno García, 1997: 100), y se hace más popular a inicios del Reino Medio, a medida que la realeza gana poder ${ }^{140}$. Los srw del texto de Rediukhnum son agentes estatales enviados a las provincias en nombre de la administración central; y el propio Rediukhnum se define como un $s r$ (1. 16) y como un $d m i$ i $s r w$ "compañero de oficiales" (1. 4), criado en palacio y enviado a trabajar a Dendera (1.10-11), y claramente muestra cercanía a la realeza (1. 18-19). Sin embargo, estos $s r w$, al igual que los $h q 3 w h w t$, están únicamente relacionados directamente con la reina, no con el rey. 
Tal y como se ve en el texto de Rediukhnum, los srw eran enviados desde la capital a las provincias para tener un mayor control administrativo (Moreno García, 2007: 322) ${ }^{141}$. Sus funciones allí eran colaborar en la administración de las provincias, ya sea en la recaudación de impuestos o fuerza de trabajo ${ }^{142}$, e imponer sanciones administrativas o penales (Pirenne, 1933-1935 I: 176, 206; II: 162-164, 188-189, 248; Bárta, 2013: 167). En los decretos de Coptos B y C de Pepy II se aprecian perfectamente las labores administrativas que tenían los $s r w$ en las provincias ${ }^{143}$, y en la biografía de Uni se ve como este, en calidad de $s r$, es enviado a presidir un juicio contra la esposa real ${ }^{144}$. También, al igual que los $h q 3 w h w t$, tal y como se aprecia en las Enseñanzas para Merikare, parece que los $s r w$ eran enviados por motivos militares y defensivos ${ }^{145}$. En definitiva, los srw engloban una serie de categorías de funcionarios de alta responsabilidad ligadas a palacio y con autoridad en las provincias (Moreno García, 1997: 108-109); según A. Moret (1916: 386), la actuación de estos funcionarios en las provincias durante el Reino Antiguo es un síntoma de una fuerte centralización.

\section{Conclusiones}

A finales de la VI dinastía, tanto los $h q 3 w h w t$ como los srw eran oficiales íntimamente relacionados con el estado. Por un lado, la $h w t$, tal y como se ve en la biografía de Ibi, era una circunscripción territorial controlada por el estado con funciones administrativas, de almacenaje y de defensa, y su propósito era que la capital tuviera control sobre el territorio provincial y sus recursos; y por otro lado, los $s r w$, bien documentados en varios periodos históricos, eran altos oficiales con altos cargos y responsabilidades que trabajaban directamente para el estado. Entre sus funciones principales estaban las de recaudar impuestos o fuerza de trabajo en las provincias. Si se tienen en cuenta estos patrones de la VI dinastía, es factible creer, después de analizar la estela de Rediukhnum, que estos reyes tebanos de principios de la XI dinastía tuvieron la intención de reinstaurar el control estatal sobre las provincias, el cual se perdió durante el Primer Periodo Intermedio. Sin embargo, tanto Rediukhnum, en calidad de $s r$ o agente real educado en la corte, como los $h q 3 w h w t$ y los $s r w$, son enviados directamente por la reina, sin que el rey sea, siquiera, mencionado en todo el texto. Aunque otros textos de principios de la XI dinastía, como el de Djari, vincule al $h q 3$ h $h w t$ con el rey, la biografía de Rediukhnum parece estar evidenciando una realidad distinta en la que las competencias que solían pertenecer exclusivamente al rey ahora pueden ser de otros individuos. La reina, en este caso, podría representar estos poderes locales que se generaron durante el Primer Periodo Intermedio, que gozaron de una gran autonomía y que expresaron iniciativas políticas, económicas y culturales. Parece que los reyes tebanos intentaron reinstaurar la administración del Reino Antiguo, pero estos nuevos focos de poder frenaron estas aspiraciones. De tal forma, estos reyes se vieron forzados a pactar con estas familias locales y a aceptar otras medidas alternativas, como la restructuración de algunos cargos como el de imy-r3 pr o la sustitución de la ḩwt por la hnrt en el Reino Medio.

El principal propósito de estas medidas administrativas en las provincias era el suministro de comida y materias primas a palacio. Aunque Rediukhnum
141. Un pasaje de las Instrucciones del visir representa muy bien esta idea: $s m i=s n n=f h p r t n b(t) m_{-}{ }^{r}=s n$ “Ellos (=los srw) le informaron (al visir) de todo lo que ocurría bajo su cargo" (Instrucciones del Visir R22).

142. En egipcio existe un sutantivo que refleja la función del $s r: \mid$ $\infty \beta_{0}^{\infty}$ puesto", "reclutamiento", etc. (Wb IV: 193, 8; HL1: 729). Un ejemplo en el Decreto de Coptos B de Pepy II: ir imy-r\} šm $m^{`} w$ irt $\{=f\}$ $s r w=s n$ "En cuanto al supervisor del Alto Egipto que recauda sus impuestos" (Museo de El Cairo, JE 41893, I. 9; Urk. I: 280-283, núm. 171; Strudwick, 2005: 107-109, núm. 24); además de un verbo: $1 \odot$ M sri "ser oficial" (Edel, 1955: 185-186, núm. 425; 231, núm. 497; Wb IV: 189, 13; HL1: 727). Un ejemplo muy aislado en el decreto de Coptos M de Neferkauhor (VIII dinastía): iw wd. $n h m(=i) s r r=f$ "Mi majestad ha ordenado que él sea nombrado oficial" ("Ubicación desconocida", I. 7; Urk. I: 300-301, núm. 176E; Strudwick, 2005: 121, núm. 35).

143. Museo de El Cairo, JE 41893; Museo de El Cairo, JE 41491; Urk. I: 284-288, núm. 172; Strudwick (2005: 109-111, núm. 25).

144. šn.t(w) ht m ipt-nswt r hmt nswt wrt hts $m$ sšts rdi $h m=f h 3 y=f r$ sdm $w^{e} . k(w) n w n . t(w)$ t3yty s3b t3ty $n b$ sr $n b$ im $w p-r(=i) w^{c} \cdot k(w)$ "Se indagó una cosa en el harén real contra la esposa real, la grande, favorita en secretos. Su majestad hizo que yo fuera para escuchar estando yo solo. No hubo ningún visir ni ningún oficial allí a parte de mí estando yo solo" (Museo de El Cairo, JE 6304/CGC 1435, col. 10-11).

145. $[t r] i \operatorname{sr} w(=k) s w \underline{d} 3 r m \underline{t}=k$ srwd $t 3 \check{s}(w)=k p h r(w) t=k$ "Respeta a tus oficiales, cuida a tu gente y haz fuerte tus fronteras y tus patrullas" (Enseñanzas para Merikare $\mathrm{P}_{3} 8$ -

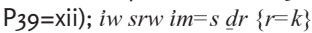
hrw hnw smn $t 3 \check{s}(w)$ “Los oficiales están en ella (=la zona fronteriza) desde el día de la residencia. Las fronteras están listas" (Enseñanzas para Merikare $\left.\mathrm{P}_{98}-\mathrm{P}_{102}=x x x v i\right)$. 
no menciona ningún tipo de tributación, tal y como sí lo hace Henenu, sí que muestra preocupación por enviar a la reina lo producido en su hacienda. Por otro lado, otro de los objetivos más relevantes era la defensa de las fronteras del reino tebano de principios de la XI dinastía. Tanto los $h q 3 w h w t$ como los $s r w$, tal y como se ve, principalmente, en documentación de la VI dinastía, podían tener funciones militares. Estos documentos muestran que una de las funciones principales de la $h w t$ era actuar como centro de almacenaje para proveer a las expediciones militares que partían de aquí. A su vez, los $h q 3 w$ $h w t$ también participaban en estas expediciones y las $h w w t$ se consideraban auténticas fortalezas. No parece aventurado suponer que estas funciones pudieran mantenerse en época de Rediukhnum, precisamente un periodo de guerra civil entre Tebas y Heracleópolis. Textos contemporáneos como el de Djari muestran a un $h q 3$ 3 $h w t$ que va a la guerra y al rey Intef II Wahankh que trata a las $h w w t$ como centros defensivos. De hecho, la misma hacienda de Rediukhnum habría podido actuar como centro de avituallamiento de estas expediciones militares.

\section{Notas}

90 Tanto Neferu I como Neferu Il se encuentran bien documentadas: la primera se encuentra documentada en la estela de Tjetji (Museo Británico, EA 614, l. 1) y en la de Djari

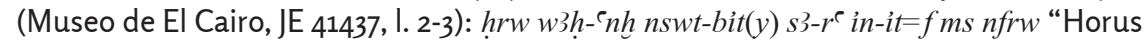
Wahankh, señor de las dos tierras, hijo de Re Intef, nacido de Neferu"; y la segunda, como madre de Intef III, en la estela de Tjetji (Museo Británico, EA 614, l. 13) y en el dintel del santuario de Heqaib, en Elefantina (Elefantina, Santuario de Heqaib 10o, I. 1; Habachi, 1985 I: 111, fig. 3; II: lám. 190): hr nht-nb-tp-nfr nswt-bit(y) s3-r in in-it=fms nfrw "Horus Nekhetnebtepnefer, señor de las dos tierras, hijo de Re Intef, nacido de Neferu". En una estela fragmentada (posiblemente de Rediukhnum) se lee lo siguiente: [...] $n n s w t s 3-r^{c}$ $i n$ - $i t=f n m(w) t=f n f r w$ "[...] del rey, hijo de Re Intef, de su madre Neferu" (Museo de la Universidad de Pensilvania, "sin número de inventario"; Hayes, 1971: 477 n. 4; Postel, 2004: 315, núm. 50). Como no da el nombre nswt-bity del rey es imposible averiguar si se trata de Intef II Wahankh o Intef III. El mismo problema ocurre en 3 trozos de lino encontrados en depósitos del templo de Mentuhetep II: $s 3-r^{\ulcorner}$in-it= $f(3)$ ms nfrw "hijo de Re Intef el grande, nacido de Neferu (Postel, 2004: 316-317, núm. 53-55). (En página 61.)

96 Inehbi (Mentuhetep II) aparece en el ataúd de la princesa Aashyt como imy-r3 $p r$ de su hacienda privada (Museo de El Cairo, JE 47267, I. $\mu .2$; Clère y Vandier, 1948: 25-29); Intef (Mentuhetep II) en un caso similar con la princesa Kauit (Museo de El Cairo, JE 47397, col. w; Clère y Vandier, 1948: 31-32); y el propio Rediukhnum con la reina Neferukayet (I. 11-12). Incluso, para la XIII dinastía, esta tradición continúa tal y como se ve en la estela de Pesesh, quien se define como imy-r3 pr $n$ hmt nsw "imy-r' $p r$ de la esposa real” (Museo de Antigüedades de Leiden, Inv. Cat. 17; Boeser, 1909: lám. xvi). Un ejemplo de haciendas no pertenecientes a la familia real en la XI dinastía en la estela de Tjebu, quien afirma servir $m$ [imy]-r3 pr $n h q^{`} w$ 6 "como [imy]-r3 pr para 6 gobernadores" (Museo de El Cairo, CGC 20005, I. 7; Lange y Schäfer, 1902-1925 i: 4-5; iv: lám. i; Clère y Vandier, 1948: 2-3, núm. 3; Landgráfová, 2011: 52-53, núm. 16). (En página 62.)

101 La llamada "escena de las cuentas" de la tumba de Niankhkhnum y Khnumhetep (Niuserre) muestra a 4 hombres con el título de $h q 3 w$ niwwt $t 3$ - $m h w$ " "jefes de las ciudades del Bajo Egipto" ante el imy-r $r$ pr, acompañada de la siguiente inscripción: $s \check{s} m$ it tht h̆ $h 3$ bdt 
tnt šnt it dì (n) imy-r’ pr "Informe de la cebada. Pago de 1000 (sacos) de trigo y pago de 100 (sacos) de cebada entregados (al) imy-r3 pr" (Abusir norte, tumba de Niankhkhnum y Khnumhetep, I. sz.28.4; Moussa y Altenmüller, 1977). (En página 62.)

102 La recitación 571 de los Textos de los ataúdes ( qd hww imyt mw “Construcción de una hwt que está en el agua") enumera las tareas más importantes del imy-r’ pr: iw $\operatorname{skr}$ hnt $(y)$

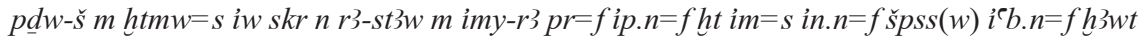
$n h w t=f t n$ "Sokar, el que está al frente de Pdjuesh es su tesorero. Sokar de Rastau es su imy-r’ pr. Él ha contabilizado sus bienes. Él ha traído riquezas y ha llenado la capilla del altar de este, su hwt" (CT VI: 172). Aunque, en este caso, los protagonistas son los dioses, estos deben estar adoptando roles administrativos basados en modelos egipcios reales. (En página 62.)

106 Nakhty posee el cargo de imy-r3 c $b$ nb "Supervisor de todas las cabezas de ganado" (Chatsworth, I. 1; Müller, 1933: 165-206) y Henenu (Mentuhetep II) el de imy-r3 “ $b$ "Supervisor de las cabezas de ganado" (MMA, Inv. Cat. 26.3.217, I. 4; Hayes, 1949: 43-49, fig. 1, lám. 4), mientras Khety se define como ip r3-idr $n \check{s} m^{`} w$ "El que censa las cabezas de ganado del Alto Egipto” (Museo de El Cairo, JE 45057, I. 4-5; Gardiner, 1917: 33-35, lám. xiii; Schenkel, 1965: 282, núm. 476; PM I: 617; Landgráfová, 2011: 54-55, núm. 17). Ya con Amenemhat I, Intefiqer, hijo de Mentuhetepiqer, porta el título de imy-r3 tst "Supervisor de las cabezas de ganado" (Museo Británico, EA 1628, I. 1-3; Budge, 1911-1922 V: lám. 1-2; Bell, 1971: 15; Franke, 2007: 149-174, fig. 1-3; Landgráfová, 2007: 286-287, núm. 64). (En página 63.)

112 Itibi de Asiut se vanagloria de haber asegurado la frontera heracleopolitana con el reino tebano por medio de htmw "fortalezas" (Asiut III/N12.1, col. 35) mientras Neheri menciona una ith $m$ - $h r-i b w$ "fortaleza en medio del distrito" (Hatnub 20, col. 13). También merece la pena recordar referencias en las Enseñanzas para Merikare: qd hwwt $m$ t3-mhw nn šri rn $n$ s $m$ irt $n=f n$ h d. $. n . t w$ niwt grg.ti qd hwwt "Construye $h w w t$ en el Bajo Egipto. El nombre de un hombre no será pequeño por lo que ha hecho. Una ciudad no se destruye estando ella (bien) construida" (Enseñanzas para Merikare P106-P109=xxxviii). (En página 64.)

$113 \mathrm{Al}$ igual que los reyes de Heracleópolis, los reyes tebanos también modificaron el paisaje por medio de fortalezas. Tal es el caso de Intef, hijo de Tjefi (Mentuhetep II), que dice: iw grt rdi.n w(i) $n b=$ i $m n^{\top} r t$ [hntt] $m$ imy-r\} hnrt "Mi majestad ciertamente me posicionó en (el nomo de) Naret[khnetet] (=Heracleópolis) como supervisor de la fortaleza" (MMA, Inv. Cat. 57.95, I. 1-col. 2); o del propio Intef II Wahankh: $q d . n(=i) h w w t-n t r$ " "He construido templos" y wn.n(=i) ith (w) "He inaugurado fortalezas" (Museo de El Cairo, JE 2101/CGC 20512, col. 2-3). También se envían imyw-r mše "comandantes” como Ip (El-Saff, tumba de Ip, pared de la izquierda, col. 7) para controlar estas fronteras. (En página 64.)

115 En la recitación 571 de los Textos de los ataúdes (qd ḩwt imyt mw “Construcción de una $h w t$ que está en el agua") se aprecia muy bien esta red al decir de la hwt que iw ḩ3w m prw=sn "miles son sus prw" (CT VI: 171). Pero el ejemplo más ilustrativo sobre esta circulación del tributo se encuentra en la carta de Herkhuf (VI dinastía): i $w$ in $w d w n$

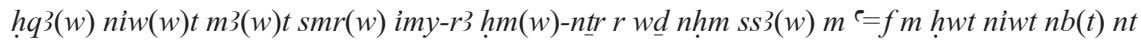
$p r$-šn $n^{\ulcorner} m h w t$ tr $n b(t)$ "Mandatos han sido traídos para los $h q 3 w$ de las ciudades vecinas, amigos y supervisores de sacerdotes para que manden suministrar provisiones de su mano, de todas las hwt de ciudades pertenecientes a un centro de producción y de todos los templos" (Qubbet el-Hawa 34, I. 25-26; Urk. I: 128-131, núm. 76; PM V: 237; Lichtheim, 1973: 26-27; Serrano Delgado, 1993: 77-79, núm. 14; Strudwick, 2005: 331-333, núm. 241). (En página 64.)

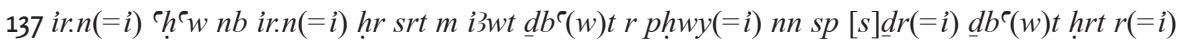
$\underline{d r} r[d i] t(=i) m$ sr nn sp s3t(=i) nn $s p[h] n r t(=i) h t n b \underline{d} d t r(=i) m-b 3 h$ srw pr.n $(=i) h r=s m$ $h t p$ sk $h r(. w) h r d \underline{d} d w d r b 3 q(=i)$ im $m-b 3 h$ srw “Yo pasé todo (este) tiempo que yo pasé sobre el consejo de oficiales en la ocupación del sello hasta mi final. No sucedió que yo dejara el sello lejos de mí desde que soy oficial, no sucedió que yo bajara mi guardia 
y no sucedió mi encarcelamiento. En cuanto a toda cosa dicha en mi contra en frente de los oficiales, yo (siempre) me libré de ello con éxito mientras caía la culpa sobre los acusadores antes de que mi inocencia fuera probada allí, ante los oficiales (Meir D2, pared de la izquierda, col. 1-4; Blackman, 1924: 24-26, lám. iv; Urk. I: 221-223, núm. 140; PM IV: 254-255; Lichtheim, 1988: 19, núm. 5; Strudwick, 2005: 369-371, núm. 270); ir.n(=i) ch`w nb ir.n $n(=i) m$ wnwt sr sk w(i) hr irt bw-nfr hr d dd mrrt n-mrwt sbt qd(=i) hr ntr "Yo pasé todo (este) tiempo que yo pasé en el cargo de oficial haciéndolo lo mejor posible y hablando de forma querida con el fin de llevar mi reputación ante el dios" (Meir D2, pared de la derecha, col. 4-5). (En página 66.) 


\section{Q Bibliografía}

» Abdalla, A. (1993). Two Monuments of Eleventh Dynasty Date from Dendera in the Cairo Museum, en: Journal of Egyptian Archaeology 79: 248-254.

»Allen, J. P. (2003). The High Officials of the Early Middle Kingdom, en: Strudwick, N. y Taylor, J. H. (eds.), The Theban Necropolis: Past, Present and Future. Londres, British Museum: 14-28.

" Allen, T. G. (1921). The Story of an Egyptian Politician, en: American Journal of Semitic Languages and Literatures 38: 55-62.

" Anthes, R. (1928). Die Felseninschriften von Hatnub: nach den Aufnahmen Georg Möllers (Untersuchungen zur Geschichte und Altertumskunde Ägyptens 9). Leipzig, J. C. Hinrichs.

»Baer, K. (1960). Rank and Title in the Old Kingdom: The Structure of the Egyptian Administration in the Fifth and Sixth Dynasties. Chicago, The University of Chicago Press.

» Barbotin, Ch. y Clère, J. J. (1992). L'inscription de Sésostris ler à Tôd, en: Bulletin de l'Institut français d'archéologie orientale 91: 1-32.

»Bárta, M. (2013). Kings, Viziers, and Courtiers: Executive Power in the Third Millennium B.C., en: Moreno García, J. C. (ed.), Ancient Egyptian Administration (Handbuch der Orientalistik 104). Leiden, Brill: 153-175.

»Bell, B. (1971). The Dark Ages in Ancient History. I. The First Dark Age in Egypt, en: American Journal of Archaeology 75: 1-26.

»Bennett, J. (1962). A New Interpretation of B.M. Stela 1203, en: Journal of Egyptian Archaeology 48: 159-160.

»Blackman, A. M. (1924). The Rock Tombs of Meir, vol. IV: The Tomb-Chapel of Pepionkh the Middle, Son of Sebkhopte and Perkhernefert (D, no. 2) (Archaeological Survey of Egypt 25). Londres, Egypt Exploration Fund.

» Blackman, A. M. (1931). The Stele of Thethi, Brit. Mus. No. 614, en: Journal of Egyptian Archaeology 17: 55-61.

»Blackman, A. M. (1935). The Stela of Nebipusenwosret: British Museum No. 101, en: Journal of Egyptian Archaeology 21: 1-9.

"Boeser, P. A. A. (1909). Beschreibung der Aegyptischen Sammlung des Niederländischen Reichsmuseums der Altertümer in Leiden, vol. I: Die Denkmäler der Zeit zwischen dem Alten und Mittleren Reich und des Mittleren Reiches. Parte I: Stelen. La Haya, Martinus Nijhoff.

» Boorn, G. P. F. van der. (1988). The Duties of the Vizier: Civil Administration in the Early New Kingdom. Londres-Nueva York, Kegan Paul International.

»Borchardt, L. (1937-1964). Denkmäler des Alten Reiches (ausser den Statuen) im Museum von Kairo, nr. 1295-1808. CGC. 2 vols. Berlín, Reichsdruckerei.

»Breasted, J. H. (1906). Ancient Records of Egypt: Historical Documents from the Earliest Times to the Persian Conquest, vol. I: The First to the Seventeenth Dynasties. Chicago, The University of Chicago Press.

» Brovarski, E. J. (1973). An Unpublished Stele of the First Intermediate Period in 
the Oriental Institute Museum, en: Journal of Near Eastern Studies 32: 453-465.

»Brunner, H. (1937). Die Texte aus den Gräbern der Herakleopolitenzeit von Siut mit Übersetzung und Erläuterungen (Ägyptologische Forschungen 5). GlückstadtNueva York, J. J. Augustin.

» Buck, A. de y Gardiner, A. H. (1935-1961). The Egyptian Coffin Texts, 7 vols. Chicago, University of Chicago Press. [=CT]

» Budge, E. A. W. (1911-1922). Hieroglyphic Texts from Egyptian Atelae, Ec., in the British Museum, 6 vols. Londres, Longmans \& Co.

» Budge, E. A. W. (1914). Egyptian Sculptures in the British Museum. Londres, Longmans \& Co.

» Černý, J. (1961). The Stela of Merer in Cracow, en: Journal of Egyptian Archaeology 47: 5-9.

»Clère, J. J. y Vandier, J. (1948). Textes de la première période intermédiaire et de la Xlème dynastie (Bibliotheca aegyptiaca 10). Bruselas, Fondation Égyptologique Reine Élisabeth.

»Daressy, G. (1907). Fragments de stèles de la XI ${ }^{\mathrm{e}}$ Dynastie, en: Annales du Service des Antiquités de l'Égypte 8: 242-247.

»Davies. N. de G. (1902). The Rock Tombs of Deir el Gebrâwi (Archaeological Survey of Egypt 11, 12), 2 vols. Londres, Egypt Exploration Fund.

»Davies, N. M. y Gardiner, A. H. (1915). The Tomb of Amenemhet (no. 82) (Theban Tomb Series 1). Londres, Egypt Exploration Fund.

»Drenkhahn, R. (1976). Bemerkungen zu dem Titel hhkr.t nswt, en: Studien zur altägyptischen Kultur 4: 59-67.

»Edel, E. (1955). Altägyptische Grammatik, vol. 1 (Analecta orientalia 34). Roma, Pontificium Institutum Biblicum.

»Erman, A. y Grapow, H. (1926-1931). Wörterbuch der ägyptischen Sprache, 5 vols. Leipzig-Berlín, Akademie Verlag. [=Wb]

» Faulkner, R. O. (1962). A Concise Dictionary of Middle Egyptian. Oxford, Griffith Institute-Ashmolean Museum. [=FCD]

» Fischer, H. G. (1959). An Example of Memphite Influence in a Theban Stela of the Eleventh Dynasty, en: Artibus Asiae 22: 240-252.

» Fischer, H. G. (1960). The Inscription of İn-İt.f, Born of Tूfi, en: Journal of Near Eastern Studies 19: 258-268.

»Fischer, H. G. (1968). Dendera in the Third Millennium B.C., Down to the Theban Domination of Upper Egypt. Locust Valley, J. J. Augustin.

» Fischer, H. G. (1996). The Tomb of I'p at El Saff. Nueva York, MMA.

"Fischer, H. G. (1997). Egyptian Titles of the Middle Kingdom: a Supplement to Wm. Ward's Index. Nueva York, MMA.

»Franke, D. (2003). The Middle Kingdom Offering Formulas: A Challenge, en: Journal of Egyptian Archaeology 89: 39-57.

»Franke, D. (2007). The Good Shepherd Antef (Stela BM EA 1628), en: American Journal of Archaeology 93: 149-174.

» Gardiner, A. H. (1917). Tomb of a Much-Travelled Theban Official, en: Journal of Egyptian Archaeology 4: 28-38. 
» Gardiner, A. H. (1947). Ancient Egyptian Onomastica, vol. 1. Londres, Oxford University Press.

" Gardiner, A. H. (1960). The Kadesh Inscriptions of Ramesses II. Oxford, Griffith Institute.

» Gayet, A. J. (1889). Musée du Louvre: stèles de la Xlle Dynastie (Sciences Historiques et Philologiques 68). París, F. Vieweg.

» Goedicke, H. (1960). The Inscription of Dmỉ, en: Journal of Near Eastern Studies 19: 288-291.

"Grajetzki, W. (2013). Setting a State Anew: The Central Administration from the End of the Old Kingdom to the End of the Middle Kingdom, en: Moreno García, J. C. (ed.), Ancient Egyptian Administration (Handbuch der Orientalistik 104). Leiden, Brill: 215-258.

" Grdseloff, B. (1948). Reseña de: Clère, J. J. y Vandier, J. (1948). Textes de la première période intermédiaire et de la Xlème dynastie (Bibliotheca aegyptiaca 10). Bruselas, Fondation Égyptologique Reine Élisabeth, en: Bibliotheca orientalis 5: 159-161.

» Griffith, F. L. (1889). The Inscriptions of Siûtț and Dêr Rîfeh. Londres, Trübner \& co.

» Griffith, F. L. (1927). The Abydos Decree of Seti I at Nauri, en: Journal of Egyptian Archaeology 13: 192-208.

» Habachi, L. (1985). Elephantine IV: The sanctuary of Heqaib, 2 vols. (Archäologische Veröffentlichungen 33). Maguncia, P. von Zabern.

» Hannig, R. (1997). Die Sprache der Pharaonen: grosses Handwörterbuch ÄgyptischDeutsch (2800-950 v. Chr.) (Hannig-Lexica 1. Kulturgeschichte der antiken Welt $64), 2^{\underline{a}}$ ed. Maguncia, Philipp von Zabern. [=HL1]

" Hayes, W. C. (1949). Career of the Great Steward Henenu under Nebhepetrē' Mentuhotpe, en: Journal of Egyptian Archaeology 35: 43-49.

" Hayes, W. C. (1953). The Scepter of Egypt: A Background for the Study of the Egyptian Antiquities in the Metropolitan Museum of Art, vol. 1. Nueva York, MMA.

"Hayes, W. C. (1971). The Middle Kingdom in Egypt. Internal History from the Rise of the Heracleopolitans to the Death of Ammenemes III, en: Edwards, I. E. S., Gadd, C. J. y Hammond, N. G. L. (eds.), Early History of the Middle East (The Cambridge Ancient History 1, parte 2), $3^{\underline{a}}$ ed. Cambridge, Cambridge University Press: 464-522.

» Helck, W. (1975). Domänenvorsteher, en: Helck, W. y Otto, E. (eds.), Lexikon der Ägyptologie, vol. 1. Wiesbaden, Harrassowitz: 1120.

» Helck, W. (1977). Die Lehre für König Merikare (Kleine ägyptische Texte 6). Wiesbaden, Harrassowitz.

" Helck, W. (1986). Titel und Titulaturen, en: Helck, W., Otto, E. y Westendorf, W. (eds.), Lexikon der Ägyptologie, vol. 6. Wiesbaden, Harrassowitz: 596-601.

» Jansen-Winkeln, K. (1988). Bemerkungen zur Stele des Merer in Krakau, en: Journal of Egyptian Archaeology 74: 204-207.

" Jones, D. (2000). An Index of Ancient Egyptian Titles, Epithets and Phrases of the Old Kingdom (British Archaeological Reports, International Series 866). Oxford, Archaeopress.

» Junker, H. (1929-1955). Gîza: Bericht über die von der Akademie der Wissenschaften in Wien auf gemeinsame Kosten mit Dr. Wilhelm Pelizaeus unternommenen 
Grabungen auf dem Friedhof des Alten Reiches bei den Pyramiden von Gîza (Denkschriften der kaiserlichen Akademie der Wissenschaften in Wien, philos.hist. Kl. 69-75), 12 vols. Viena, Hölder-Pichler-Tempsky.

»Kamâl, A. (1909). Tables d'offrandes, vol. 1. CGC. El Cairo, IFAO.

» Kees, H. (1933). Kulturgeschichte des Alten Orients: Ägypten. Munich, Beck.

» Kitchen, K. A. (1975-1990). Ramesside Inscriptions: Historical and Biographical, 8 vols. Oxford, Blackwell. [=KRI]

»Koch, R. (1990). Die Erzählung des Sinuhe (Bibliotheca aegyptiaca 17). Bruselas, Fondation égyptologique reine Elisabeth.

» Kruchten, J-M. (1981). Le décret d'Horemheb. Bruselas, Editions de l'Université de Bruxelles.

» Lacau. P. (1904). Sarcophages antérieurs au Nouvel empire, vol. 1. CGC. El Cairo, IFAO.

"Landgráfová, R. (2007). Topic-Focus Articulation in Biographical Inscriptions and Letters of the Middle Kingdom (Dynasties 11 - 12). Tesis doctoral inédita. Univerzita Karlova v Praze, Praga.

"Landgráfová, R. (2011). It is my Good Name that you should Remember. Egyptian Biographical Texts on Middle Kingdom Stelae. Praga, Charles University, Faculty of Arts, Czech Institute of Egyptology.

"Landgráfová, R. (2015). In the Realm of Reputation: Private Life in Middle Kingdom Auto/Biographies, en: Miniaci, G. y Grajetzki, W. (eds.), The World of Middle Kingdom Egypt (2000-1550 BC): Contributions on Archaeology, Art, Religion, and Written Sources (Middle Kingdom Studies 1). Londres, Golden House Publications: 177-185.

» Lange, H. O. (1896). Zwei Inschriften der Fürsten von Hermonthis, en: Zeitschrift für ägyptische Sprache und Altertumskunde 34: 25-35.

»Lange, H. O. y Schäfer, H. (1902-1925). Grab- und Denksteine des Mittleren Reichs im Museum von Kairo. CGC. 4 vols. Berlín, Reichsdruckerei.

» Lapp, G. (1986). Die Opferformel des Alten Reiches: unter Berücksichtigung einiger späterer Formen (Sonderschrift des deutschen archäologischen Instituts, Abteilung Kairo 21). Maguncia, P. von Zabern.

» Lichtheim, M. (1973). Ancient Egyptian Literature: a Book of Readings, vol. I: The Old and Middle Kingdoms. Berkeley-Londres, University of California Press.

» Lichtheim, M. (1988). Ancient Egyptian Autobiographies Chiefly of the Middle Kingdom: a Study and an Anthology (Orbis biblicus et orientalis 84). Friburgo, Universitätsverlag; Gotinga, Vandenhoeck \& Ruprecht.

»Limme, L. (1979). Stèles égyptiennes (Musées royaux d'Art et d'Histoire de Bruxelles 4). Bruselas, Musées royaux d'art et d'histoire.

» Madkour, H. (2016). An Eleventh Dynasty Stela of the Priest Ka-whmi, en: Studien zur altägyptischen Kultur 45: 217-225.

"Mariette, A. (1869-1880). Abydos: description des fouilles executées sur l'emplacement de cette ville. París, F. Vieweg.

"Morgan, J. de y otros (1894). Catalogue des monuments et inscriptions de l'Égypte antique, vol. I: De la frontière de Nubie à Kom Ombo. Viena, A. Holzhausen.

» Moreno García, J. C. (1996). Administration territoriale et organisation de 
l'espace en Egypte au troisième millénaire avant J.-C.: grgt et le titre '(n)d-mr grgt, en: Zeitschrift für ägyptische Sprache und Altertumskunde 123: 116-138.

» Moreno García, J. C. (1997). Études sur l'administration, le pouvoir et l'idéologie en Égypte, de l'Ancien au Moyen Empire (Aegyptiaca leodiensia 4). Lieja, C.I.P.L.

" Moreno García, J. C. (1999a). Hwt et le milieu rural égyptien du Ille millénaire: économie, administration et organisation territoriale (Bibliothèque de l'École des hautes études Sciences historiques et philologiques 337). París, H. Champion.

" Moreno García, J. C. (1999b). J'ai rempli les pâturages avec des vaches tachetées... Bétail, économie royale et idéologie en Egypte, de l'Ancien au Moyen Empire, en: Revue d'égyptologie 50: 241-257.

"Moreno García, J. C. (2007). State, Rural Landscape, and Domestic vs. Institutional Agriculture in the 3rd Millennium, en: Bollig, M. y otros (eds.), Aridity, Change and Conflict in Africa. Proceedings of an International ACACIA Conference Held at Königswinter, Germany October 1-3, 2003 BC (Colloquium Afrikanum 2). Colonia, Heinrich-Barth-Institut in Kooperation mit Universität zu Köln: 313-330.

» Moreno García, J. C. (2009). El Primer Período Intermedio, en: Parra Ortiz, J. M. (coord.), El Antiguo Egipto. Sociedad, economía, política. Madrid, Marcial Pons: 181-208.

" Moreno García, J. C. (2013). The Territorial Administration of the Kingdom in the 3rd Millennium, en: Moreno García, J. C. (ed.), Ancient Egyptian Administration (Handbuch der Orientalistik 104). Leiden: Brill: 85-151.

» Moreno García, J. C. (2014). Les échanges entre l'Egypte et les regions voisines (2100-180o avant J.-C.), en: Égypte, Afrique and Orient 75: 7-18.

" Moreno García, J. C. (2016). Social Inequality, Private Accumulation of Wealth, and New Ideological Values in Late 3 rd Millennium BC Egypt, en: Meller, H. H., Hahn, H. P., Jung, R. y Rish, R. (eds.), Rich and Poor - Competing for Resources in Prehistoric Societies: 8th Archaeological Conference of Central Germany, October 22-24, 2015 in Halle (Saale) (Tagungen des Landesmuseums für Vorgeschichte Halle 14). Haale, Landesamt für Denkmalpflege und Archäologie SachsenAnhalt, Landesmuseum für Vorgeschichte: 491-512.

» Moret, A. (1916). L'administration locale sous l'ancien empire égyptien, en: Comptes rendus de l'Académie des inscriptions et belles-lettres 60: 378-386.

» Mostafa, M. F. (1984-1985). Erster Vorbericht über einen Ersten Zwischenzeit Text aus Kom el-Koffar. Teil I, en: Annales du Service des Antiquités de l'Égypte 70: 419-429.

»Mostafa, M. F. (1987). Kom el-Koffar. Teil II: Datierung und Historische Interpretation des Textes B, en: Annales du Service des Antiquités de l'Égypte 71: 169-184.

» Moussa, A. H. y Altenmüller, H. (1977). Das Grab des Nianchchnum und Chnumhotep (Archäologische Veröffentlichungen 21). Maguncia, Philipp von Zabern.

» Müller, H. W. (1933). Die Totendenksteine des Mittleren Reiches, ihre Genesis, ihre Darstellungen und ihre Komposition, en: Mitteilungen des deutschen archäologischen Instituts, Abt. Kairo 4: 165-206.

" Musacchio, T. (2006). An Epigraphic Reanalysis of Two Stelae from First Intermediate Period Dendera in the Cairo Museum, en: Antiguo Oriente: Cuadernos del Centro de Estudios de Historia del Antiguo Oriente 4: 69-9o. 
»Musacchio, T. (2008). Texts and Iconography of Autobiographic Stelae Found at Dendera Dating from the First Intermediate Period, en: Bulletin of the Egyptian Museum 5: 51-74.

»Parkinson, R. B. (1991). The Tale of the Eloquent Peasant. Oxford, Griffith InstituteAshmolean Museum.

"Parkinson, R. B. (1997). The Tale of Sinuhe and Other Ancient Egyptian Poems, 1940-1640 BC. Oxford, Clarendon Press.

»Petrie, W. M. F. (1900). Dendereh 1898 (Memoir of the Egypt Exploration Fund 17). Londres, Egypt Exploration Fund.

»Petrie, W. M. F. (1909). Qurneh (British School of Archaeology in Egypt). Londres, Bernard Quaritch.

»Piehl, K. (1886-1903). Inscriptions hiéroglyphiques recueillies en Europe et en Égypte, 3 vols. Leipzig, J. C. Hinrichs.

» Pirenne, J. (1933-1935). Histoire des institutions et du droit privé de l'ancienne Égypte, 3 vols. Bruselas, Fondation Égyptologique Reine Élisabeth.

»Polotsky, H. J. (1930). The Stela of Heka-yeb, en: Journal of Egyptian Archaeology 16: 194-199.

»Porter, B. y Moss, R. L. B. (1970-1975). Topographical Bibliography of Ancient Egyptian Hieroglyphic Texts, Reliefs, and Paintings, 7 vols., $2^{\underline{a}}$ ed. Oxford, Griffith Institute, Ashmolean Museum. [=PM]

»Posener, G. (1951). Reseña de: Stock, H. (1949). Studia Aegyptiaca, vol. I: Die erste Zwischenzeit Ägyptens: Untergang der Pyramidenzeit, Zwischenreiche von Abydos und Herakleopolis, Aufstieg Thebens (Analecta orientalia 31). Roma, Pontificium Institutum Biblicum, en: Bibliotheca orientalis 8: 165-172.

"Postel, L. (2004). Protocole des souverains égyptiens et dogme monarchique au début du Moyen Empire: des premiers Antef au début du règne d'Amenemhat ler (Monographies Reine Élisabeth 10). Bruselas, Fondation égyptologique reine Elisabeth; Turnhout, Brepols.

» Ranke, H. (1933). Die ägyptischen Personennamen, vol. 1. Glückstadt, J. J. Augustin. $[=P N]$

» Roeder, G. (1913). Aegyptische Inschriften aus den Königlichen Museen zu Berlin. Inschriften von der ältesten Zeit bis zum Ende der Hyksoszeit, vol. 1. Leipzig, J. C. Hinrichs.

» Rougé, E. (1872). Notice des monuments exposés dans la Galerie d'antiquités égyptiennes, Salle du rez-de-chaussée et palier de l'Escalier du sud-est au Musée du Louvre, $3^{\mathrm{a}}$ ed. París, Typographie Charles de Mourgues Frères.

» Redford, D. B. (1990). Egypt and Canaan in the New Kingdom. Beerseva, BenGurion University of the Negev Press.

»Redford, D. B. (1992). Egypt, Canaan, and Israel in Ancient Times. Princeton, Princeton University Press.

"Serrano Delgado, J. M. (1993). Textos para la historia antigua de Egipto. Madrid, Cátedra.

»Sethe, K. (1908-1922). Die altaegyptischen Pyramidentexte nach den Papierabdrücken und Photographien des Berliner Museums, 4 vols. Leipzig, J.C. Hinrichs. [=Pyr] 
» Sethe, K. (1933). Urkunden des Alten Reichs. Leipzig, J. C. Hinrichs. [=Urk. I]

»Sethe, K. (1935). Historisch-biographische Urkunden des Mittleren Reiches, vol. 1. Leipzig, J. C. Hinrichs. [=Urk. VII]

»Schenkel, W. (1965). Memphis, Herakleopolis, Theben: die epigraphischen Zeugnisse der 7.-11. Dynastie Ägyptens (Ägyptologische Abhandlungen 12). Wiesbaden, Harrassowitz.

» Schenkel, W. (1973). Ein Tür stürz von der Grabkapelle des Königs Wih-'nh Antef, en: Mitteilungen des deutschen archäologischen Instituts, Abt. Kairo 29: 215-220.

» Schenkel, W. (1976). Bemerkungen zu den wichtigsten Inschriften aus dem Königsfriedhof von el-Tarif, en: Arnold, D. (ed.), Gräber des Alten und Mittleren Reiches in El-Tarif (Archäologische Veröffentlichungen 17). Maguncia, P. Von Zabern: 50-59.

"Schrane, L. (1981). Egyptian Provincial Administration in the Early Middle Kingdom. Tesis doctoral inédita. University College London, Londres.

"Stock, H. (1949). Studia Aegyptiaca, vol. I: Die erste Zwischenzeit Ägyptens: Untergang der Pyramidenzeit, Zwischenreiche von Abydos und Herakleopolis, Aufstieg Thebens (Analecta orientalia 31). Roma, Pontificium Institutum Biblicum.

»Strudwick, N. D. (2005). Texts from the Pyramid Age (Writings from the Ancient World 16). Leiden-Boston, Brill.

"Swinton, J. (2012). The Management of Estates and their Resources in the Egyptian Old Kingdom (BAR International Series 2392). Oxford, Archaeopress.

»Théodoridès, A. (1965). Le Papyrus des Adoptions, en: Revue internationale des droits de l'Antiquité 12: 79-147.

» Théodoridès, A. (1969). Les ouvriers-magistrats en Egypte à l'époque ramesside (XIXe-Xxe dyn; 13e-10e s. av. J.-C.), en: Revue internationale des droits de l'Antiquité 16: 103-188.

"Troy, L. (1986). Patterns of Queenship in Ancient Egyptian Myth and History (Acta Universitatis Upsaliensis 14). Uppsala-Estocolmo, Almquist \& Wiksell International, distributors.

»Vandier, J. (1950). Mo 'alla: la tombe d'Ankhtifi et la tombe de Sébekhotep (Bibliothèque d'étude 18). El Cairo, IFAO.

»Ward, W. A. (1982). Index of Egyptian Administrative and Religious Titles of the Middle Kingdom: with a Glossary of Words and Phrases Used. Beirut, American University of Beirut.

»Willems. H. (2013). Nomarchs and Local Potentates: The Provincial Administration in the Middle Kingdom, en: Moreno García, J. C. (ed.), Ancient Egyptian Administration (Handbuch der Orientalistik 104). Leiden, Brill: 341-392. 


\section{○ Láminas}

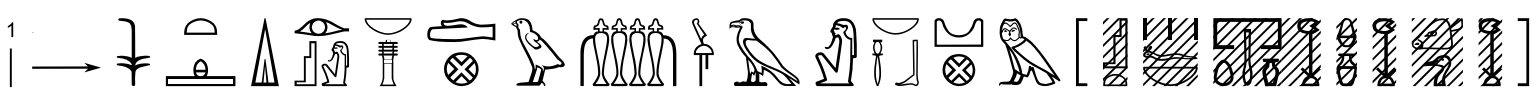

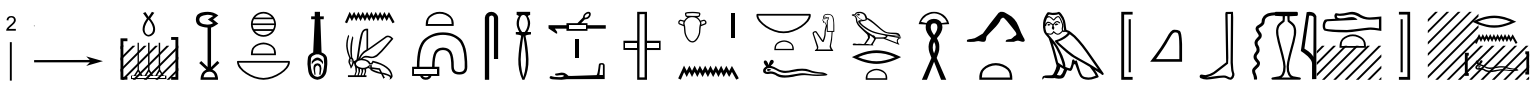

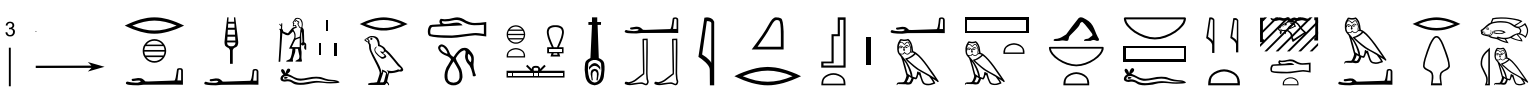

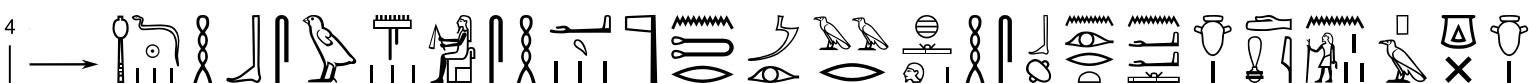

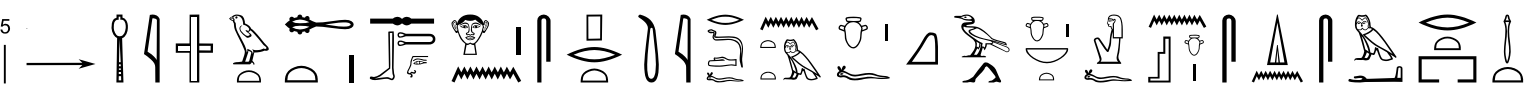

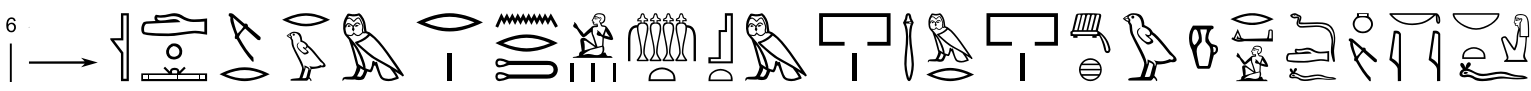
i一 I9An I $\rightarrow$ Y Un

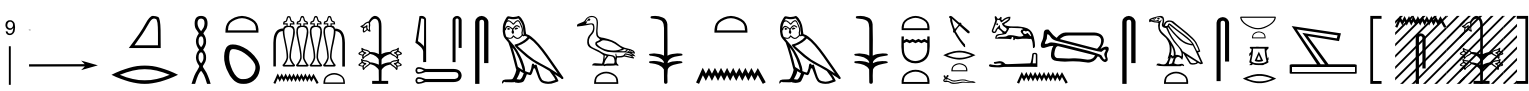

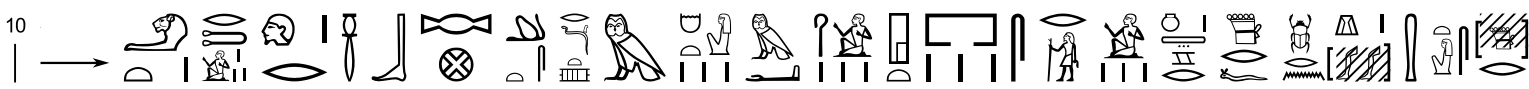

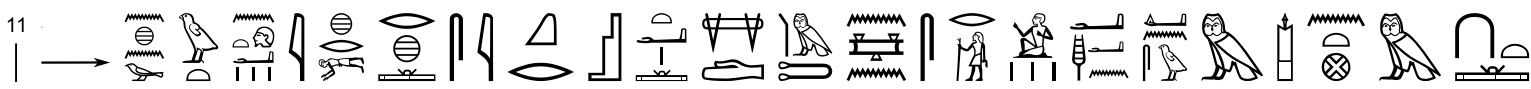

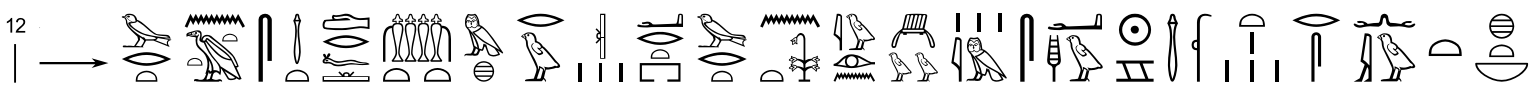

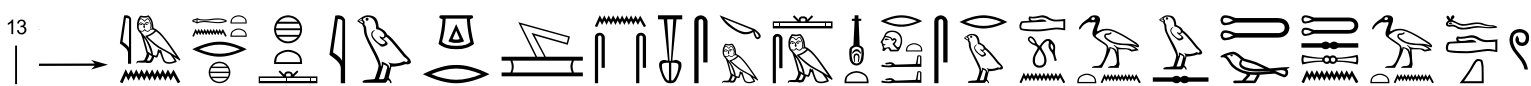

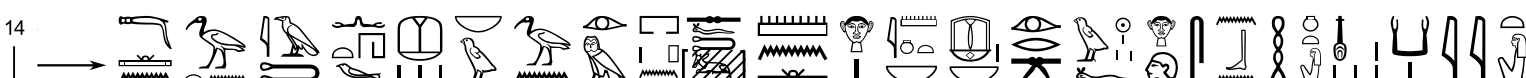
1 -

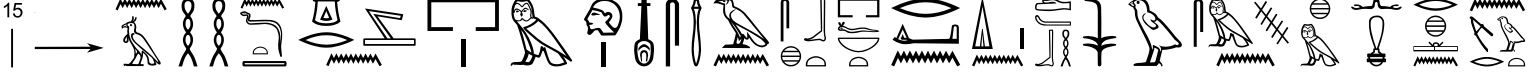

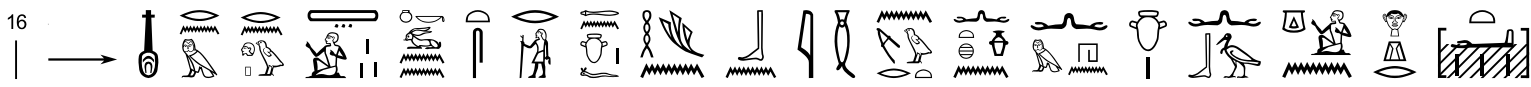

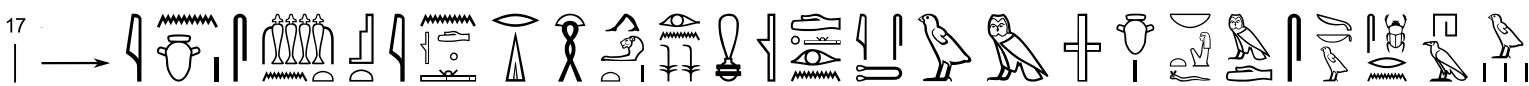

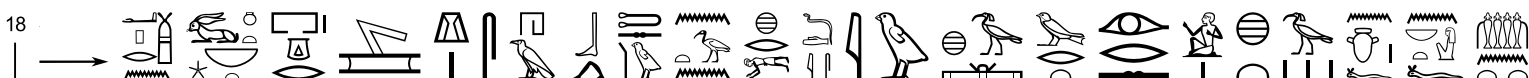

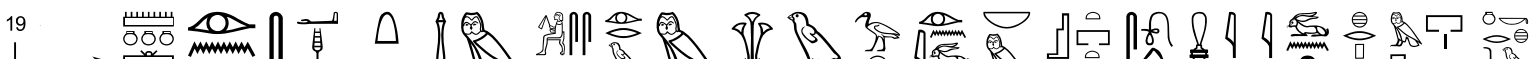
$\mid \longrightarrow \min _{2}$

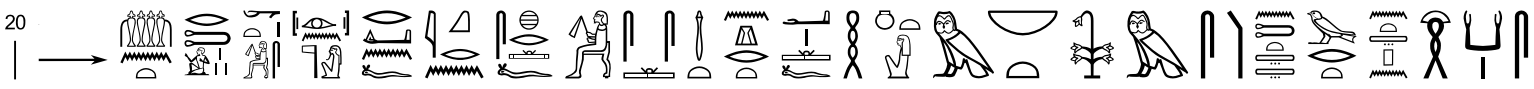

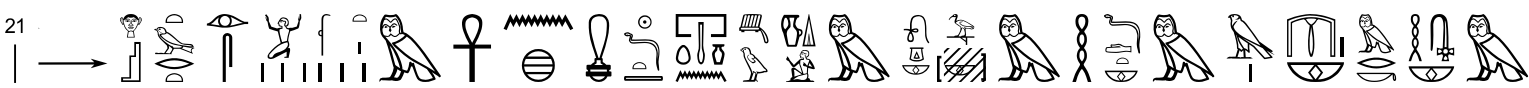

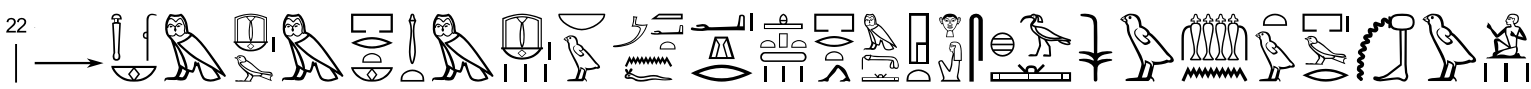

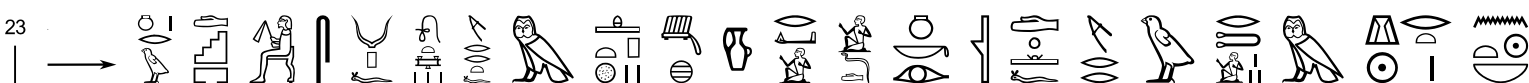




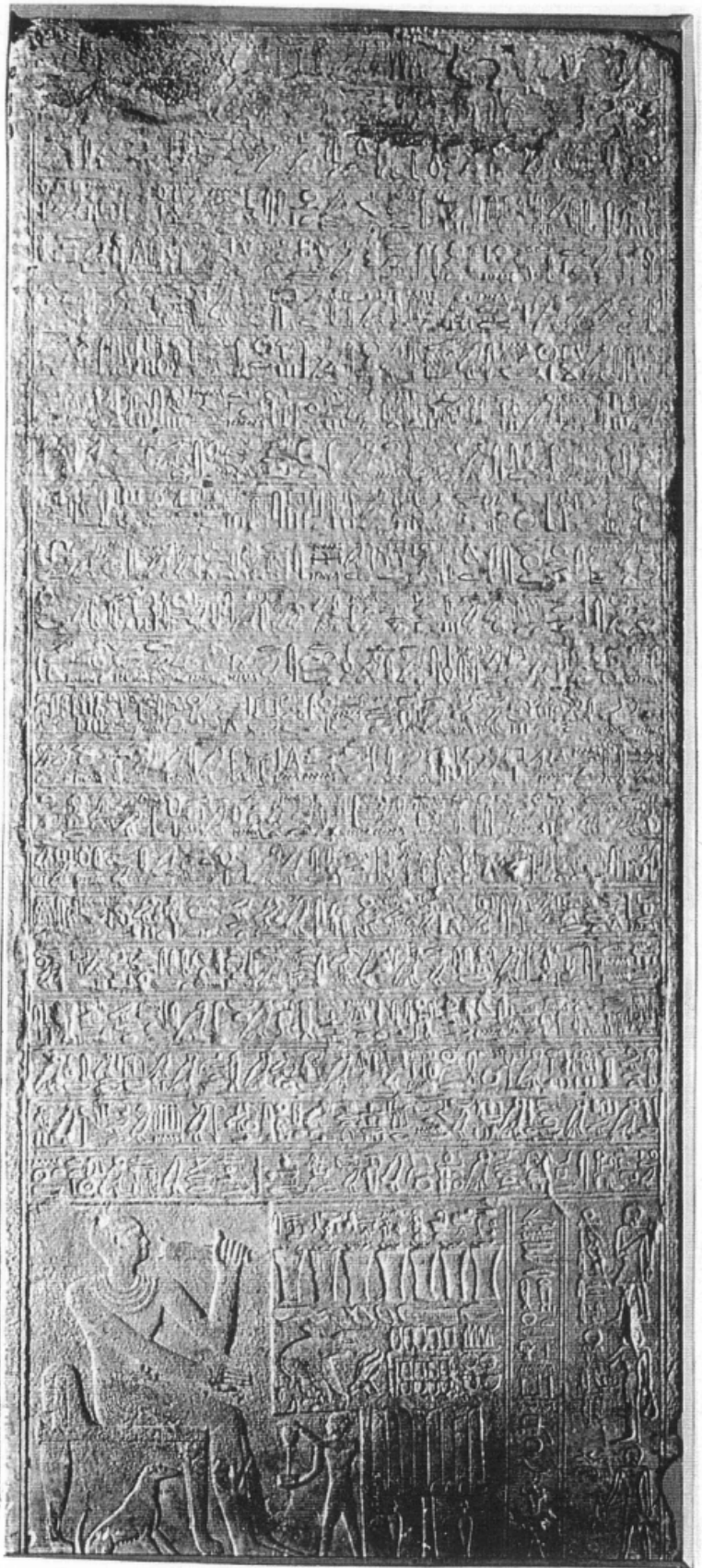

Lámina II.A Estela de Rediukhnum (JE 32138/CGC 20543). Fotografía (Lichtheim, 1988: lám. i).

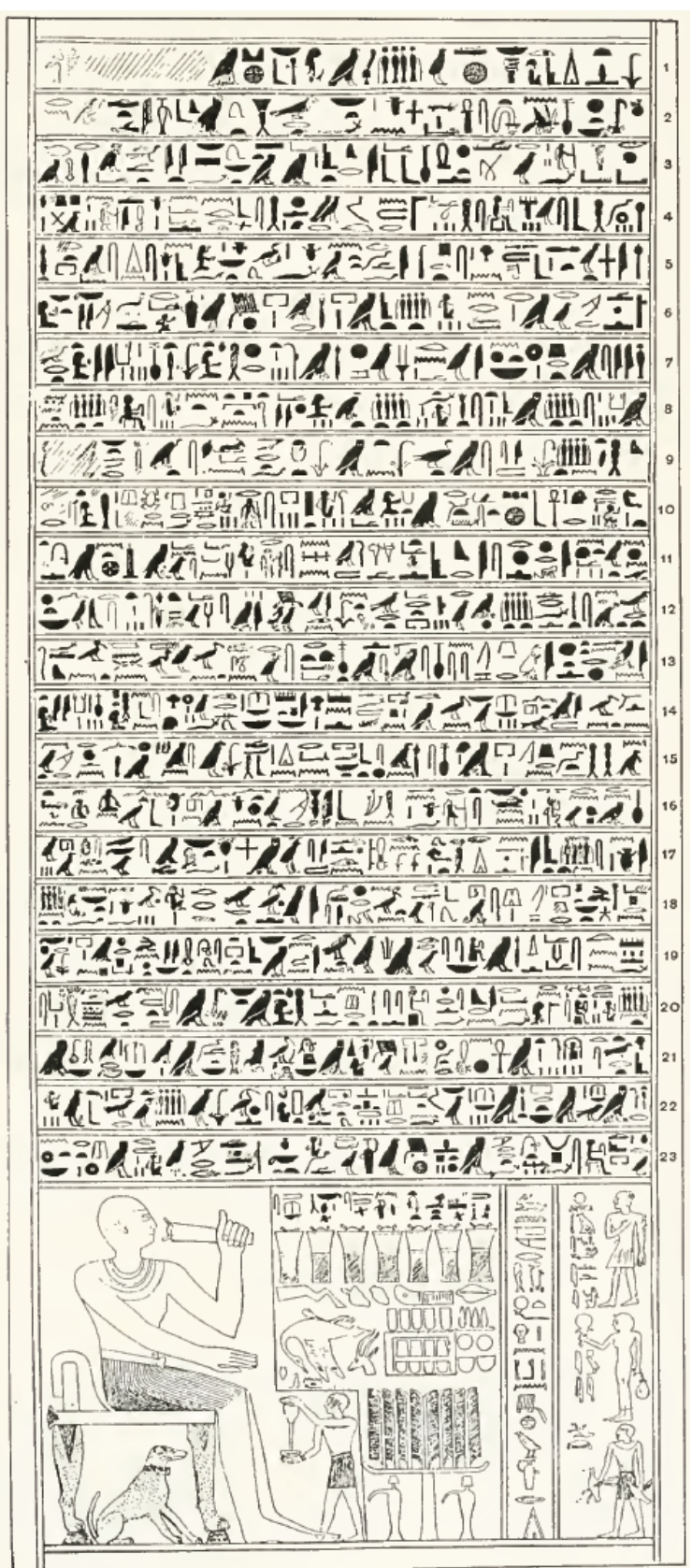

Lámina II.B Estela de Rediukhnum (JE 32138/CGC 20543). Edición de W.M.F. Petrie (Petrie, 1900: lám. xv). 
